\title{
Delivery, Installation, On-sky Verification of the Hobby Eberly Telescope Wide Field Corrector
}

\author{
Hanshin Lee ${ }^{a^{*}}$, Gary J. Hill ${ }^{a}$, John M. Good ${ }^{a}$, Brian L. Vattiat ${ }^{a}$, Matthew Shertrone ${ }^{b}$, Jerry Martin ${ }^{b}$, Emily \\ Schroeder $^{b}$, Herman Kriel ${ }^{a, b}$, Chang Jin $\mathrm{Oh}^{c}$, Eric Frater ${ }^{c}$, Bryan K. Smith ${ }^{c}$, James H. Burge ${ }^{c}$ \\ ${ }^{a}$ McDonald Observatory, University of Texas at Austin, 2515 Speedway, C1402, Austin, TX, USA 78712-0259; \\ ${ }^{b}$ Hobby-Eberly Telescope, University of Texas at Austin, 82 Mt. Locke Rd. McDonald Observatory, Texas 79734; \\ ${ }^{c}$ College of Optical Sciences, University of Arizona, 1630 E. University Blvd, Tucson, AZ
}

\begin{abstract}
The Hobby-Eberly Telescope $(\mathrm{HET})^{\dagger}$, located in West Texas at the McDonald Observatory, operates with a fixed segmented primary (M1) and has a tracker, which moves the prime-focus corrector and instrument package to track the sidereal and non-sidereal motions of objects. We have completed a major multi-year upgrade of the HET that has substantially increased the pupil size to 10 meters and the field of view to 22 arcminutes by deploying the new Wide Field Corrector (WFC), new tracker system, and new Prime Focus Instrument Package (PFIP). The focus of this paper is on the delivery, installation, and on-sky verification of the WFC. We summarize the technical challenges encountered and resolutions to overcome such challenges during the construction of the system. We then detail the transportation from Tucson to the HET, on-site ground verification test results, post-installation static alignment among the WFC, PFIP, and M1, and on-sky verification of alignment and image quality via deploying multiple wavefront sensors across 22 arcminutes field of view. The new wide field HET will feed the revolutionary new integral field spectrograph called VIRUS, in support of the Hobby-Eberly Telescope Dark Energy Experiment (HETDEX), a new low resolution spectrograph (LRS2), an upgraded high resolution spectrograph (HRS2), and later the Habitable Zone Planet Finder (HPF).
\end{abstract}

Keywords: Wide Field Corrector, Hobby Eberly Telescope, Delivery, Installation, Onsky verification

\section{INTRODUCTION}

The Hobby-Eberly Telescope (HET) is an innovative $10 \mathrm{~m}$-class telescope that operates with a fixed altitude segmented spherical primary mirror (M1) while a tracker, located at the top of the telescope, moves the prime-focus spherical aberration corrector (SAC) and instrument package in order to track the sidereal and non-sidereal motions of celestial objects. The new Wide-Field Corrector (WFC) replaces the old SAC, thereby widening the telescope instantaneous field of view (FOV) from 4 arcmin to 22 arcmin in diameter, increasing the telescope aperture from $9.2 \mathrm{~m}$ to $10 \mathrm{~m}$, and finely tuning the focal ratio to $\mathrm{f} / 3.65$ ideal for coupling optical fibers at its focal surface [1]. Much improved all-kinematic and passively athermalized opto-mechanical structures [2] ensures the structural stability of the system over the range of motions and temperatures to which the WFC is subject and the state-of-the-art protected silver reflective coatings ensures the highest telescope photon efficiency possible across the wavelength range from $360 \mathrm{~nm}$ to $1800 \mathrm{~nm}$.

The WFC's original design consisted of four reflective elements with three $\sim 1 \mathrm{~m}$ diameter concave mirrors and one $0.25 \mathrm{~m}$ diameter convex mirror, all of which are highly aspheric [2]. The WFC system was sub-contracted to the College of Optical Sciences at the University of Arizona in late 2008. Since then, there have been several major challenges in fabricating the mirror surfaces, producing a durable protected silver reflective coating, and aligning the components to the specified tolerances. Despite our best collective painstaking effort, these difficulties led to a substantial delay ( $\sim 6.5$ years versus the predicted duration of 3 years) in completing the system and also to the need of an extra aspheric corrector lens at the exit pupil of the system in order to compensate for the rather large residual spherical aberration resulted from the mirror surface fabrication challenges. Even after much more extensive alignment tests in the optics shop at the COS, these challenges imposed substantial uncertainty in the alignment state and thus imaging performance

*lee@astro.as.utexas.edu;phone 1512 471-9985; www.as.utexas.edu

$\dagger$ The Hobby-Eberly Telescope is operated by McDonald Observatory on behalf of the University of Texas at Austin, the Pennsylvania State University, Ludwig-Maximillians-Universität München, and Georg-August-Universität, Göttingen. 
of the system at the time of the delivery in late May 2015, which could only be fully verified by executing significantly elaborate on-sky multi-field wavefront sensing campaign after the installation of the system on the HET in September 2015.

To put this work in a context, we begin this paper with a summary of the challenges we encountered during the construction of the WFC system and the various methods we developed to reduce the uncertainties on the state of the system (Section 2). In Section 3, we highlight the transportation between Tucson, AZ and the HET in Fort Davis, TX and the subsequent on-site verification results. The overall performance of the transportation is detailed in terms of the shock metrology recordings and the results from the subsequent repeat of the optical system test that was conducted in Tucson before the transportation. Section 4 then details on the end-to-end telescope alignment and the on-sky verification test using the multiple Shack-Hartmann wavefront sensors (SHWFS) across the telescope FOV. These tests resulted in the WFC-only imaging performance between 0.3 and 0.8 arcsec across the field and the overall telescope imaging performance of median 1.36 arcsec including median seeing of $1.2 \operatorname{arcsec}$ and M1 stacking quality between 0.6 and 1.0 arcsec, all in the Full-Width Half-Maximum (FWHM) of a telescope Point Spread Function (PSF). This confirmed the adequate imaging performance of the WFC system and the full telescope for science instrument commissioning and normal operation.

\section{CONQUEST OF CHALLENGES}
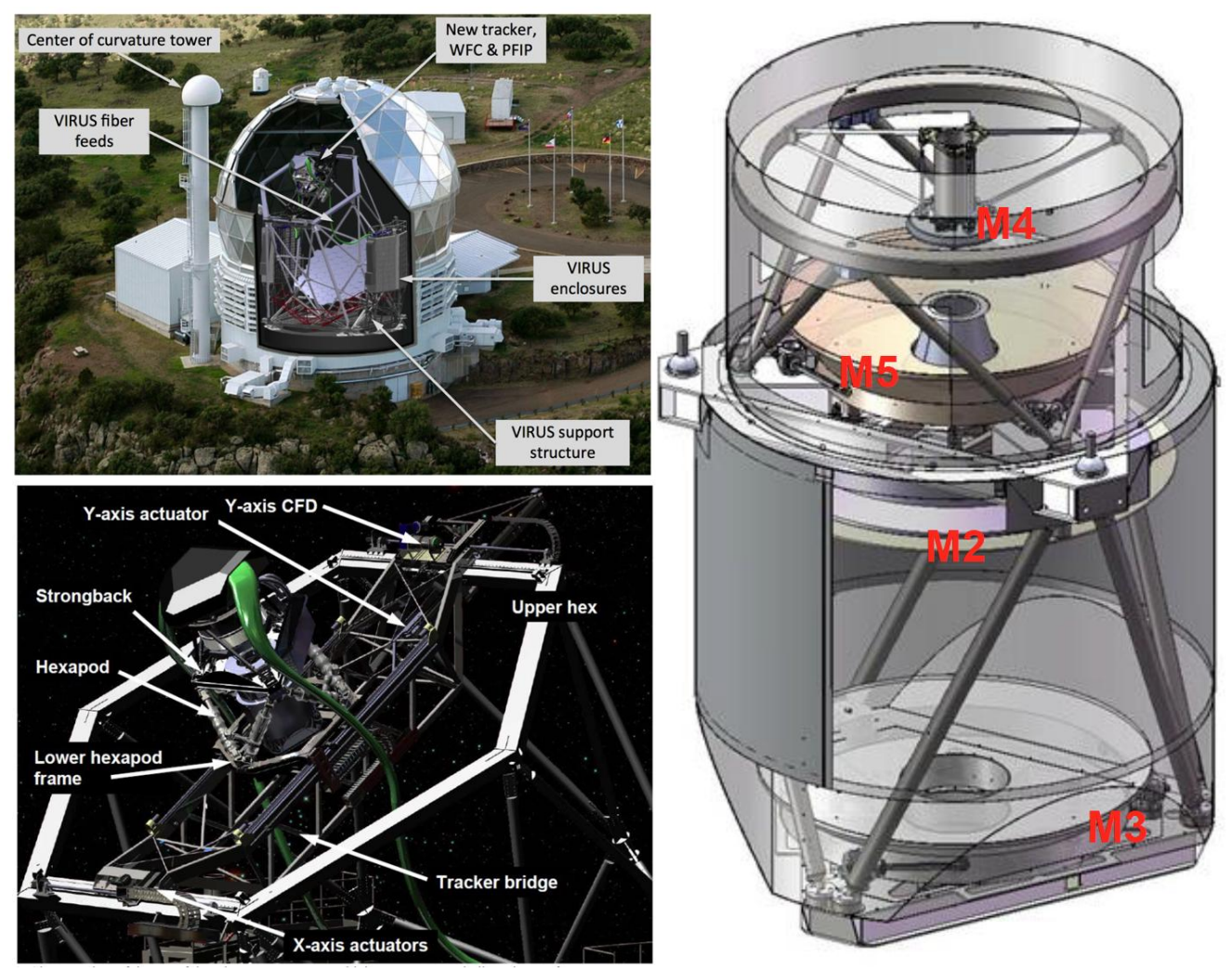

THE UNIVERSITY OF TEXAS AT AUSTIN

Figure 1 (Top left) HET with the new upgrade and the VIRUS instrument, (Bottom left) New upgraded top end of the telescope with the WFC, (Right) The WFC solid model and optical layout diagram.

As shown in Figure 1, the HET Wide-Field Upgrade (WFU) has the upgraded top end that consists of the new tracker, the WFC, and the prime focus instrument package (PFIP). On either side of the telescope, two VIRUS instrument enclosures are installed to house 75 VIRUS unit spectrographs as well as the new Low Resolution Spectrograph (LRS2)[3][4][5][6][7]. As shown on the right two panels, the WFC was originally designed as a reflective double Cassegrain system with four highly aspheric reflective surfaces. The HET's segmented primary mirror (M1) feeds beam to the WFC through the center opening of M3 as shown. The reflection from M2 forms the telescope pupil image near M3 surface. This M2-M3 pair then forms an intermediate focus on a plane between M4 and M5. The M4-M5 pair is a finite conjugate subsystem that relays the intermediate focus onto the final curved focal surface of 22-arcmin in diameter on 
sky through the system's exit pupil. The projection of the exit pupil on the 11-meter M1 corresponds to a circular disk of 10-meter in diameter, hence enlarging the HET's aperture diameter from 9.2-meter to 10-meter.

Like any other major instrumentation projects, there were numerous challenges during the course of developing the WFC system. Among those, two main challenges stand out. The first was in the fabrication of the mirror surfaces and their metrology, particularly for M3 and M5. These two mirrors have two distinct characters. M3's surface is a general even asphere and exhibits nearly $1.5 \mathrm{~mm}$ of aspheric sag departure from its best-fit sphere over a meter diameter. This amount of deviation would be normally found in much larger ( 4-meter) diameter aspheric mirrors. On the other hand, M5's surface shape is also a general asphere with the base profile of an oblate ellipsoid, which very much resembles a wide, deep, and steep salad bowl, as one might be able to make out from the optical layout in Figure 1. M5 exhibits a lesser degree of sag departure than M3's, but it is still nearly $1 \mathrm{~mm}$. Both mirrors imposed a common fabrication challenge of removing large amounts of material while keeping the mid-spatial frequency structure in check. For M5, its steepness brought an extra challenge of tool pressure reduction while polishing toward mirror edge due to large gravity vector swing going from the center to the steep edge of the optic. While M2 and M4 were relatively easier aspheres and thus fabricated without a major problem, it took nearly two years to fabricate M3 and M5, which were figured using two separate computer controlled polishing machines for grinding and polishing and each with a Swing-arm Optical CMM (SOC) surface profilometer (Figure 2).
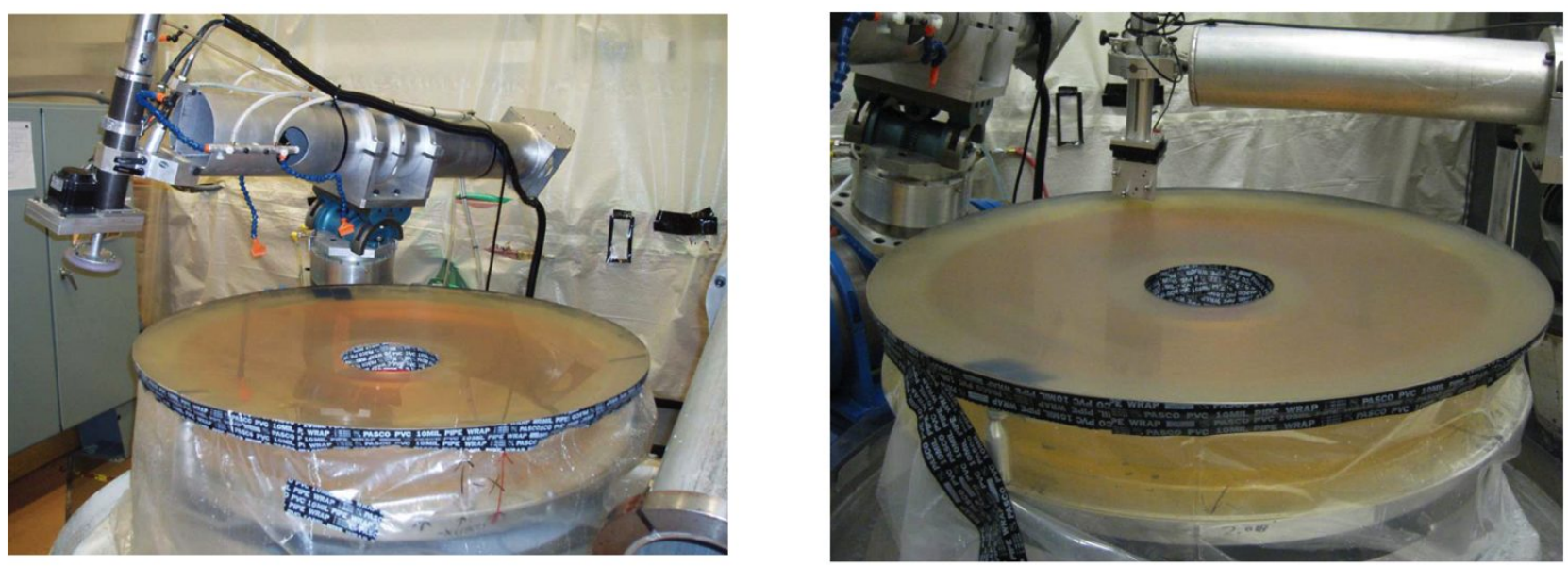

Figure 2 On the left is shown the robotic computer controlled grinder/polisher. The machine incorporates a Swing arm Optical CMM (SOC) shown on the right, which makes in situ profile measurements at several clocking angles of the part that later synthesized to estimate a two dimensional figure map. Two such machines are used; one machine performs initial shaping and polish out with highspeed grinding/polishing spindle and optical probe that measures diffuse reflections; the other machine performs final figuring with pitch laps, guided by a high performance SOC that incorporates an interferometric sensor.

The large aspheric departure of M3 and M5 added difficulties in the surface metrology. Although the SOC were able to make an accurate measurement of relatively high spatial frequency components, the instrument itself needed

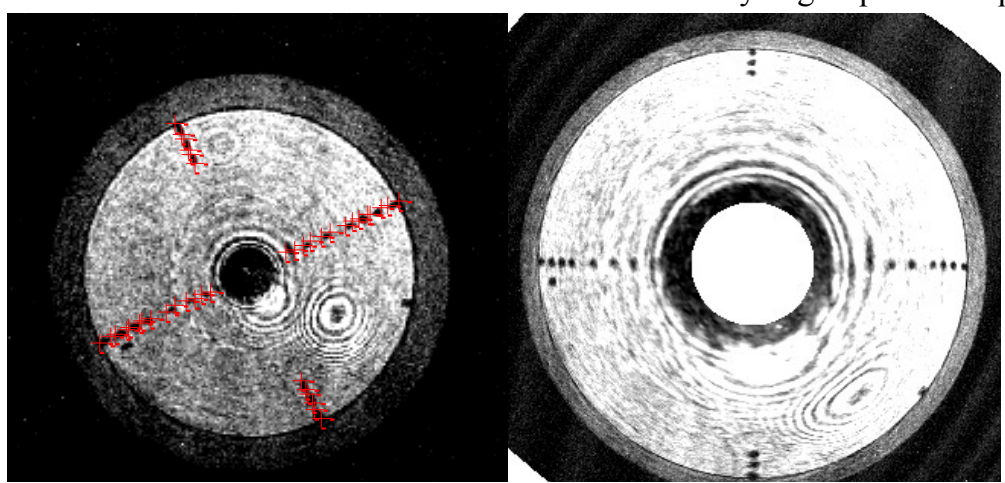

Figure 3 (Left) A raw inner annulus measurement from the M3 CGH test that is morphed into the correct annulus (Right). The fiducial markers are visible on the measurements. external metrology, specifically a laser tracker measurement of the surface radius of curvature, in order to properly reduce the raw data from the SOC. Therefore, if the laser tracker measurement were incorrect, the subsequent low order estimate from the SOC data for describing the surface prescription is also incorrect even if its high spatial frequency surface map is estimated correctly. On the hardware side, the polishing tool on the SOC arm constantly changes its vector relative to the gravity as it sweeps across the mirror surfaces. This was affected by the swing-arm bending differently over the mirror surface and thus resulted in incorrect tool vectors. For a 
mirror with shallow aspheric departure and steepness, this effect is usually insignificant, but, for M3 and M5 (more significant for M5), the aspheric departures and the steepness of the surfaces were large enough to make this effect clearly visible in the surface figure as later found out. In both M3 and M5, this effect in combination of other effects as described below exhibited as a significant amount of spherical aberration that could not be compensated by adjusting mirror separations. Although we had a redundant surface test using a Compute Generated Hologram (CGH) and an interferometer, this test also suffered from the large aspheric departure and steepness of these two mirrors. For M3, two different CGHs were needed to cover the inner and outer annuli of M3 aperture, which then stitched into one full aperture surface map. Two partial surface maps had a narrow overlapping annulus for later stitch. Each map in its raw form exhibits substantial distortion because the CGHs had to bend beam so much to map rays onto the desired area on the mirror. This distortion had to be morphed into the correct sub-aperture shape by matching the image of a series of dots laid on the mirror surface across a diameter to their known coordinates (Figure 3). This added some uncertainties in the final full aperture map. Also, since different annular zones were sampled, the CGHs had to be in different set-ups and the stitching two measurements over an overlapping narrow annulus always left artifacts that had some degree of impact on the final accuracy of the full aperture map. All these metrology challenges added up to a point where the variation of the mirror prescriptions from different measurements was too large to be acceptable, which was, very unfortunately, discovered not at the time of figuring mirrors, but much later during the system integration and test phase. It was also realized that there was inconsistency in the input data fed into analyzing the SOC metrology data that resulted in the surface prescription estimate amazingly consistent with the design values, which in reality turned out to be incorrect.

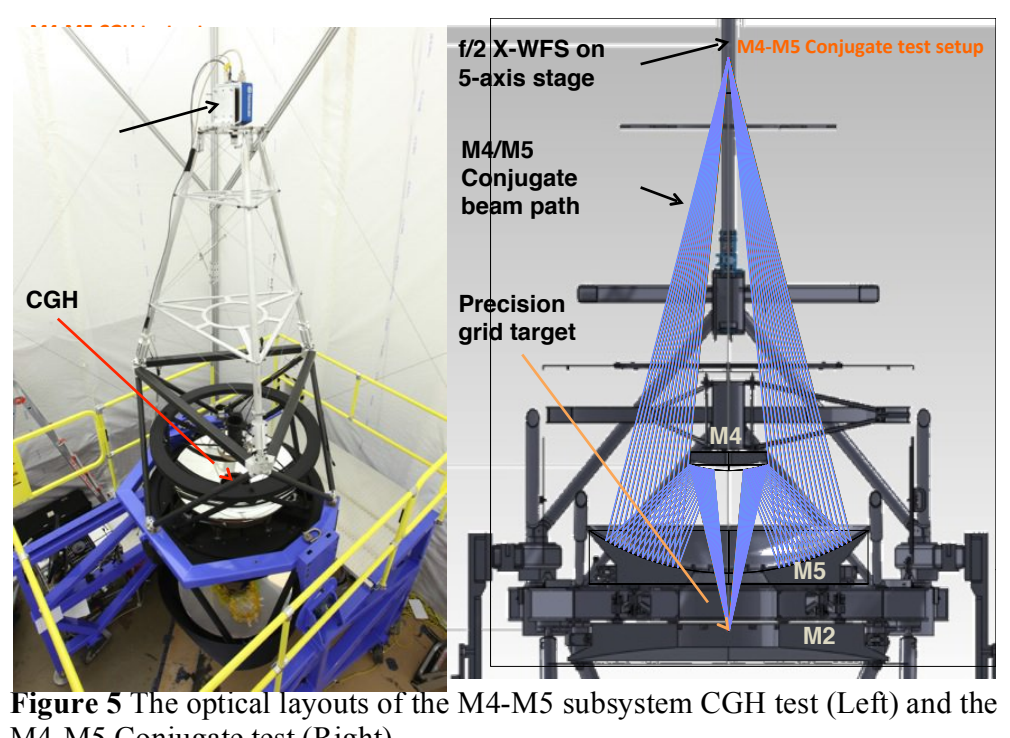
M4-M5 Conjugate test (Right).
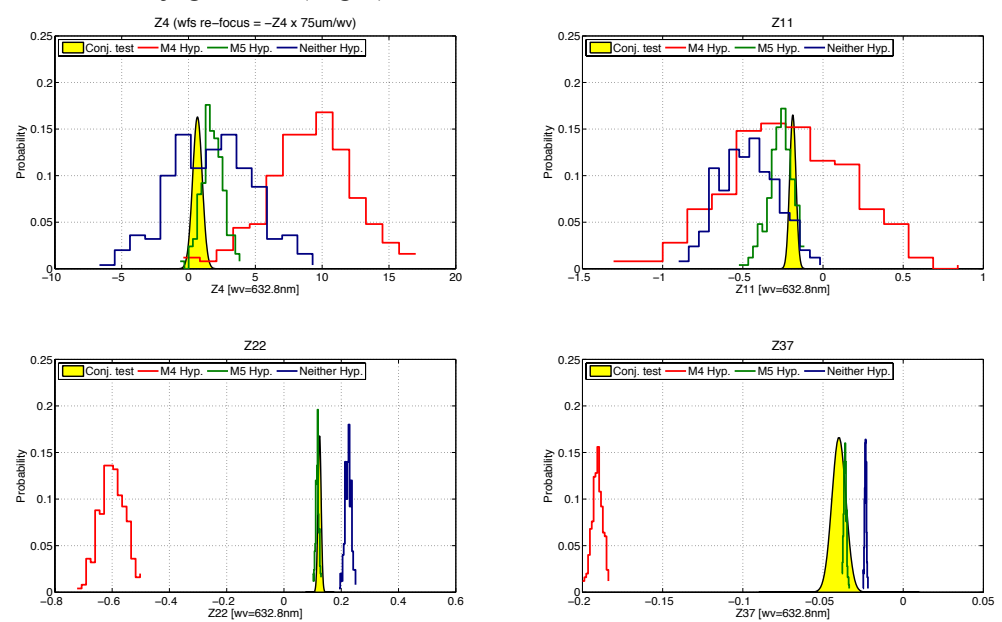

Figure 4 The main result of the M4-M5 Conjugate test indicates the test data is in agreement with M5 being the cause of the excess spherical aberration discovered in the M4-M5 CGH subsystem test.
These revelations began happening when we were executing the M4-M5 subsystem test as part of the overall alignment of the WFC system in early 2014 (in the left panel of Figure 5 and in Figure 11). This was an on-axis double-path CGH test with an interferometer. As soon as this test started producing a reasonable full-aperture interferogram, it was quickly noticed that there was significant deviations in spherical aberration and the location of the interferometer of the test from what the model predicted based on the surface metrology data. This triggered a set of investigation to understand where these deviations were coming from. We used the M4-M5 pair test itself as a way to estimating the possible M4 or M5 surface prescriptions under certain assumptions about the test set-up. Since M4 was subcontracted to an outside vendor, there was some reservation about the status of M4. However, our initial expectation was that M5 was more likely to be the cause of these anomalies, given the difficulties in fabricating and measuring the surface. In order to confirm this preliminary indication, we came up with an independent test called the M4-M5 conjugate test (in the right panel of Figure 5). This test utilized the fact that M4-M5 pair forms finite conjugate points on either side of the pair where stigmatic images can form. On the conjugate point near M5, we placed a precision grid-dot target that was illuminated by diffuse $635 \mathrm{~nm}$ red LED light. At the conjugate above M4, we installed a ShackHartmann wavefront sensor with 20 subapertures across the M4-M5 pair's pupil 
diameter in order to measure the wavefront aberration from different dots on the target through the subsystem. This is essentially a multiple field aberration measurement and was used to diagnose alignment state of this pair as to be discussed later, but for this particular application we only needed wavefront data from the very central dot of the target, i.e. on-axis, since we were only interested in the axially symmetric aberrations mainly affected by the mirror prescriptions and the test set-up. We ensured a proper alignment of the grid target fixture and the SH wavefront sensor relative to the subsystem reference and executed the test. The goal of this test was to determine whether the excess spherical aberration was coming from M4 or M5 or something else. From the test, four specific aberrations were measured. Their likelihoods for three different hypotheses were computed and then compared to their distributions from the measurements (Figure 4). The result strongly favored M5 hypothesis, confirming our initial expectation.
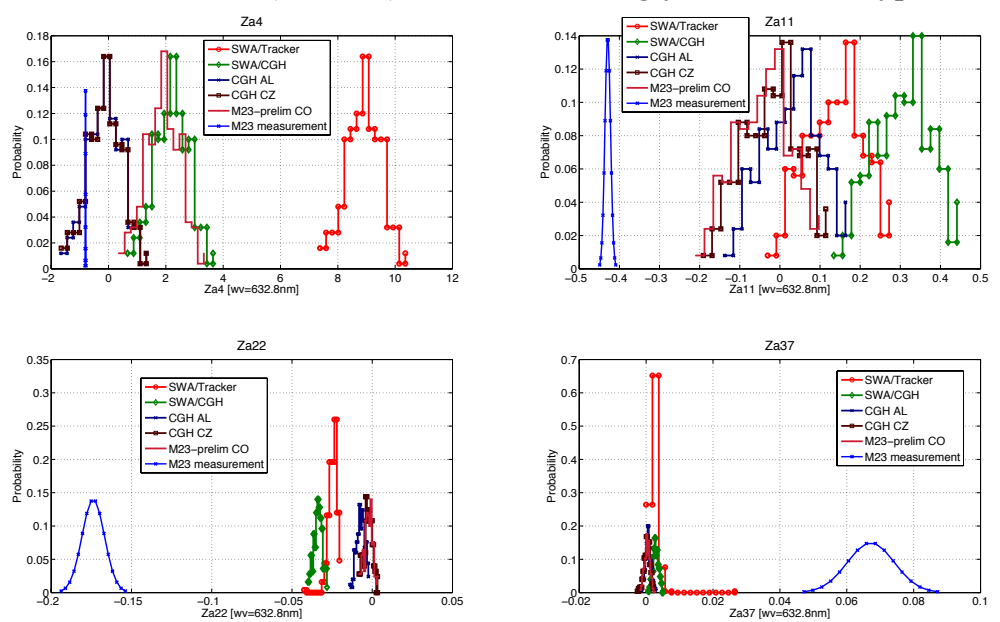

Figure 6 The M2-M3 CGH subsystem test results in terms of axially symmetric Zernike modes.

After the M4-M5 pair tests, we executed a subsystem CGH test for the M2-M3 pair. Unlike the M4-M5 pair, this subsystem does not have finite conjugate points where stigmatic images can form. However, M2 was a mild asphere and was fabricated/tested in-house and thus we had a higher confidence about the status of M2 surface and thus we had the anticipation that the M2-M3 CGH test (see Figure 11 for the optical layout) in its original layout would produce an excess spherical aberration compared to its optical model. Figure 6 shows the likelihood functions of four prescription-critical aberrations plotted for different M3 prescriptions that are based on different types of surface data. Here the nominal M2-M3 subsystem test set-up was assumed. Also plotted are the probability functions of the same aberrations measured directly from the M2-M3 test, which indicate that none of the predictions by the possible surface prescriptions matches the measurements. Also, the dispersion among the likelihoods of the existing prescriptions is quite large, particularly in the spherical aberration (Za11 in the plot), which highlights the level of uncertainty in the knowledge of the M3 prescription. We used the M2-M3 subsystem test data to come up with an additional potential surface prescription of M3. This estimation includes the uncertainties associated with the test set-up, measurement, and the knowledge of the M2 prescription. We iterated on this estimation 4 times before we get relatively stable M3 prescription estimate. Using the all direct surface metrology (i.e. SOC, Laser Tracker curvature measurement, $\mathrm{CGH}$ ) data and indirect sub-system test data (i.e. M2-M3 CGH data, M4-M5 CGH data, M4-M5 conjugate test data), we came up with the statistically most likely prescriptions for M3 and M5 among all possible scenarios (Figure 7).
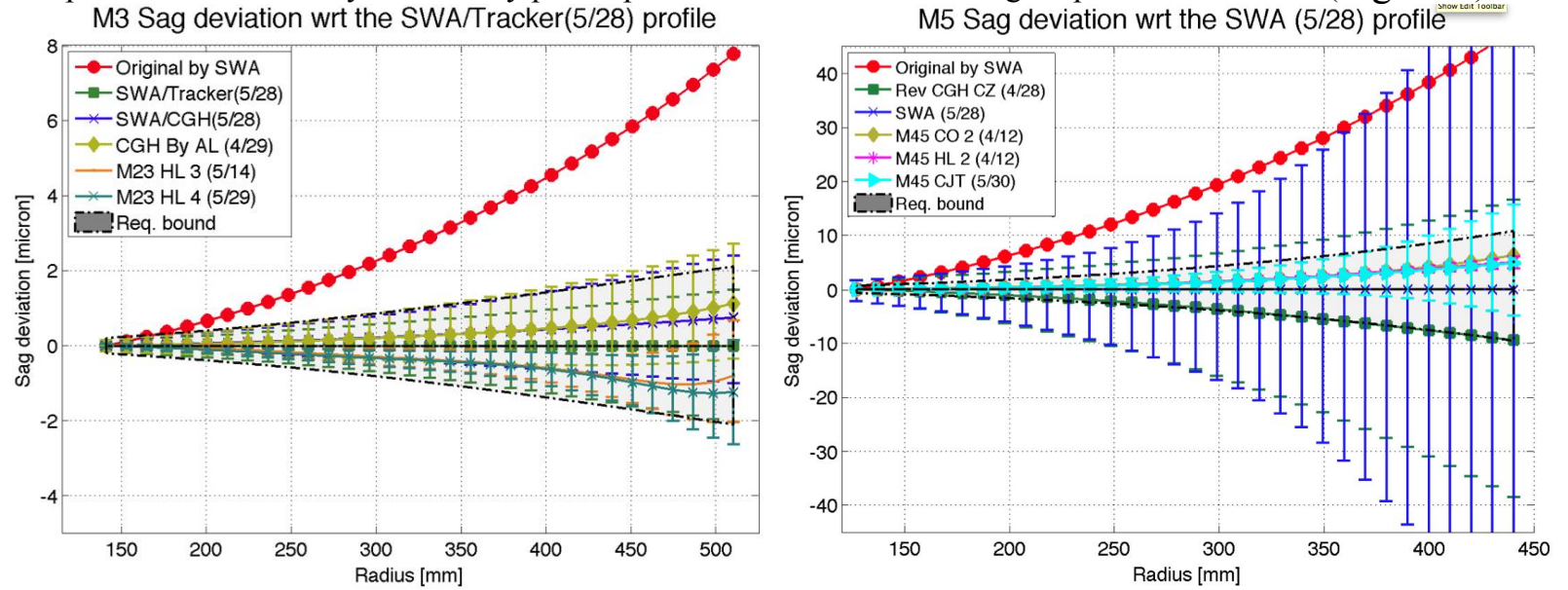

Figure 7 The radial sag profiles of possible M3 (Left) and M5 (Right) prescriptions with respect to the statistically most likely prescriptions. 
Some of the direct surface metrology data were revised and corrected for inconsistencies discovered during this investigation. As a result, all of the statistically possible prescriptions were in close agreement with each other within the required bounds as marked in the plots. The red filled circle lines highlight how far off the originally estimated M3 and M5 prescriptions were relative to what appear to be the most likely prescriptions. Unfortunately, these adopted prescriptions are too different from the original to be balanced out by adjusting the mirror separations. At the best mirror spacing, we would still have more than 1 wave rms of spherical aberration across all fields and thus fail to meet the image quality requirement. This necessitated an extra aspheric corrector lens at the exit pupil of the system. As to be discussed later, these adopted prescriptions with the extra aspheric corrector lens were then confirmed during the System CGH test (see Figure 11).

Table 1 The WFC alignment error budget and the achievable alignment accuracy using the original scheme.

\begin{tabular}{|c|c|c|}
\hline Degrees of Freedom & Alignment tolerance & Achievable alignment accuracy (RSS) \\
\hline M2 to M3 Axial & $100[\mathrm{um}]$ & $11.1[\mathrm{um}]$ \\
\hline M2 to M5 Axial & 100 [um] & 10.9 [um] \\
\hline M4 to M5 Axial & 20 [um] & 10.9 [um] \\
\hline M2 Decenter & 50 [um] & 21.6 [um] \\
\hline M3 Decenter & 50 [um] & 16.1 [um] \\
\hline M4 Decenter & 20 [um] & $12.6[\mathrm{um}]$ \\
\hline M4M5 Decenter & $50[\mathrm{um}]$ & $12.6[\mathrm{um}]$ \\
\hline M2 Tilt & $10.3[\operatorname{arcsec}]$ & $5.7[\operatorname{arcsec}]$ \\
\hline M3 Tilt & 10.3 [arcsec] & $5.7[\operatorname{arcsec}]$ \\
\hline M4 Tilt & $16.7[\operatorname{arcsec}]$ & $6.6[\operatorname{arcsec}]$ \\
\hline M4M5 Tilt & $11.7[\operatorname{arcsec}]$ & $5.7[\operatorname{arcsec}]$ \\
\hline M2M5 together decenter & 250 [um] & $22.6[\mathrm{um}]$ \\
\hline M2M5 together tilt & $58.7[\operatorname{arcsec}]$ & $7.8[\operatorname{arcsec}]$ \\
\hline
\end{tabular}

So far, we discussed the challenges related to M3 and M5 surface prescriptions. The second challenge about to be discussed was found in the alignment of the system. In Table 1, we summarize the alignment error budget and the achievable accuracy using the original alignment scheme, which is described as follows.

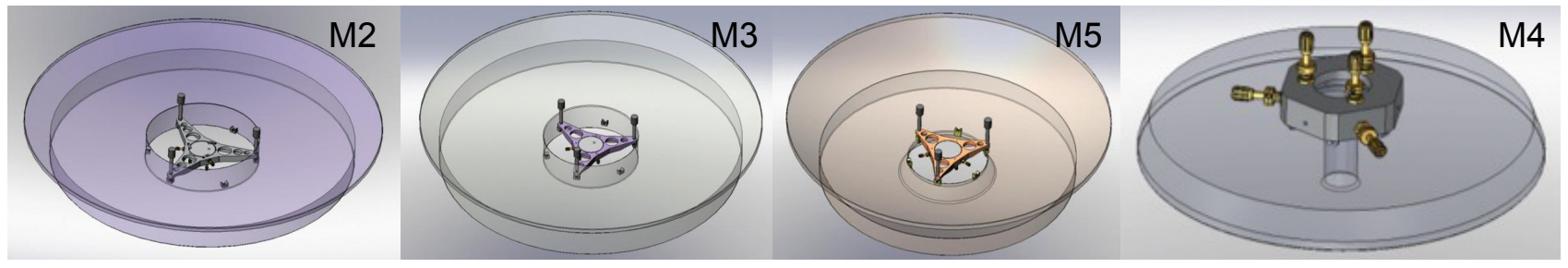

Figure 8 Solid models of M2, M3, M5, M4 with their center references.

As shown in Figure 8, each mirror has two sets of center references (CRs), for the sake of redundancy. A CR is a flat glass with a CGH pattern written on it. Each CR is registered to its corresponding mirror in 5 degrees of freedom (DOF) and become the surrogate to the mirror. Therefore, the actual alignment of the system is done via aligning these CRs with respect to each other. The CGH pattern on a CR facilitates the alignment process as to be described later. To register a $\mathrm{CR}$ to a mirror, we starts with aligning each mirror to the axis of interferometer in rotation by reducing the tip/tilt and coma aberrations. Once the mirror is aligned, a CR is installed on the kinematic V-blocks on the inner surface of the mirror central opening. A Point-Source-Microscope (PSM) on a 5-DOF stage is then mounted above the CR on a stationary mounting fixture. The PSM is then used to view a centering feature inscribed in the center of each CR as part of the CGH pattern. On rotation, this pattern rotates if the CR is decentered with respect to the mirror. Depending on the initial PSM alignment relative to the mirror, the location of this rotation center in the PSM image could be outside the field of view and thus the translation stages of the microscope are used to bring the rotation center within the PSM. Each CR has its own 5 DOF adjusters built into its cell using 5 piezo-motors. These motors are adjusted until the image of the $\mathrm{CR}$ feature stops rotating as the mirror is rotated. At that point, the CR center coincides with the optical axis of the mirror. The PSM is then changed into a mode where it projects out a collimated beam that reflects from the CR surface and forms a spot on the PSM image. Again, the PSM may not be able to capture the spot if its alignment to the mirror is off. The tip/tilt stage of the PSM is then used to bring the spot within the field of view of the microscope. If the CR 
surface is normal to the optical axis of the mirror, the spot is stationary on rotation of the mirror. And the tip/tilt adjuster on the CR is used to null out the spot rotation, at which point the CR is normal to the mirror's optical axis. Once this registration is complete, then the $\mathrm{CR}$ is bonded to its cell structure. Each $\mathrm{CR}$ has $1 / 2$-inch diameter laser tracker spherically mounted retro reflector (SMR) nests bonded to the cell structure. This makes it possible to register SMRs on these mounts to the CR in $x, y, z$ positions. Figure 9-(G) shows a CR with three $1 / 2$-inch tooling balls on the SMR mounts being registered to the CR under the PSM mounted to a precision Coordinate Measuring Machine (CMM). The centers of the tooling balls should be very close to those of precision $1 / 2$-inch SMRs. Once this registration is completed, it is possible to use a Laser Tracker (LT) in order to accurately locate the center of the CR in the 3-dimensional space and this facilitate the initial alignment of the mirrors within the capture ranges of the subsequent optical alignment instruments. Also possible is to determine the $z$ location of the CR with respect to the vertex of the mirror surface. A separate SMR fixture is placed at several locations along the outer and inner edges of each mirror. Their points in the 3D space are used to fit the mirror surface, which then locates the $x, y, z$ coordinate of the mirror vertex. Then the SMRs on the CR are measured and their relative location with respect to the vertex is determined, thereby the relative height of the $\mathrm{CR}$ to the mirror vertex is obtained.

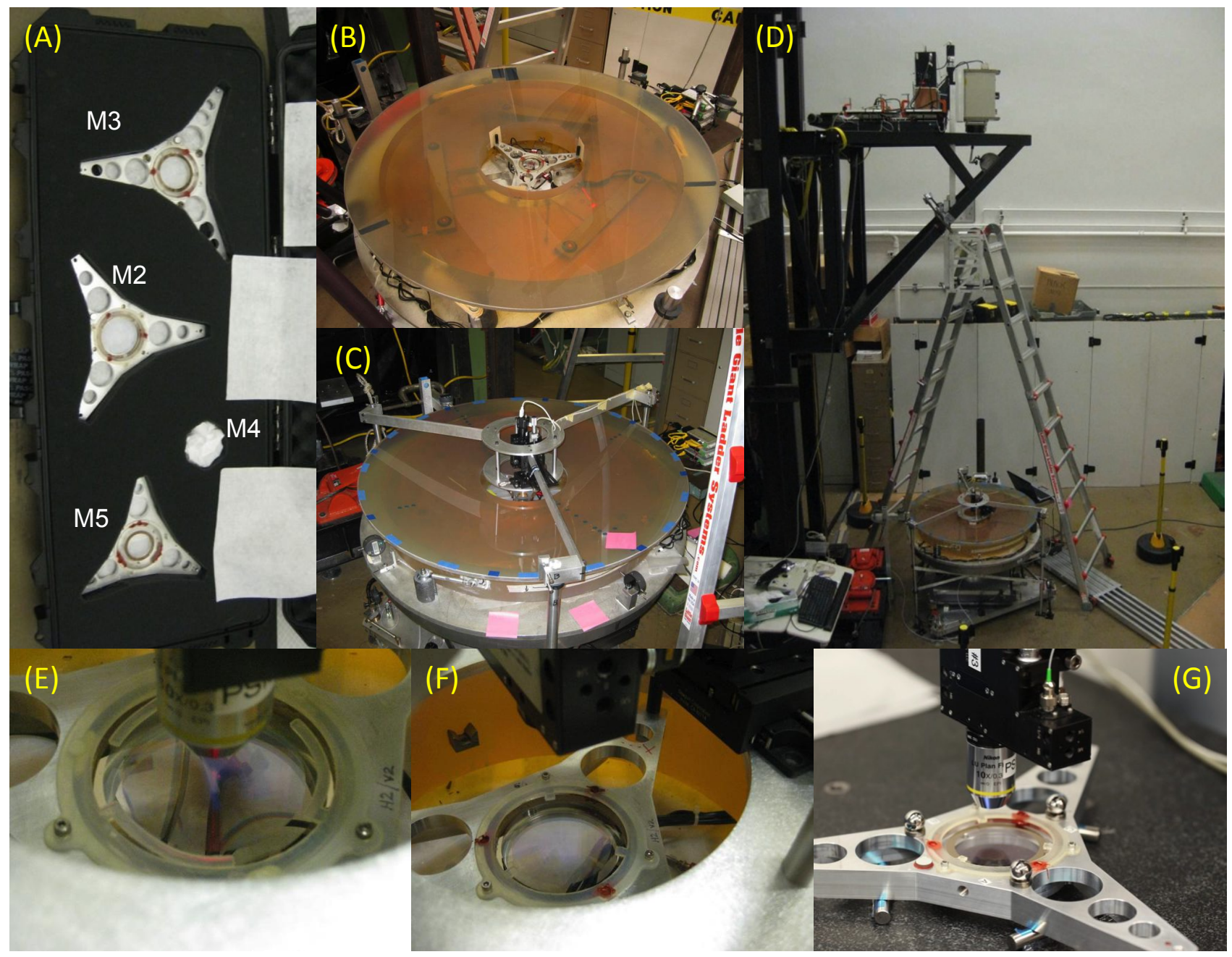

Figure 9 The Center reference to mirror registration procedures.

Unlike other mirrors' CRs, the M4 CRs do not have SMR nests glued on. This is mainly because this CR is placed on the back of M4 and thus completely obscured by the M4 support structure and M4 itself, thus preventing us from using the LT. Therefore, we adopted a scheme that uses the M4 CR as the system reference and aligns other CRs to it. To facilitate the initial LT alignment, however, we built a separate fixture that can be mounted on the back of M4 like the M4 CR, but has an extended shaft. This shaft has a SMR nest glued at the tip and thus can slide through the center opening of M4 and lets an SMR to be attached just below the M4 vertex. This SMR was registered in height to the M4 
vertex very accurately, but not in $\mathrm{X}$ and $\mathrm{Y}$. The table in Figure 10 summarizes the CR registration quality relative to the mirrors and the comparison to the required alignment tolerances.

Once the initial alignment by the LT and CR SMRs is completed, further refinement is made using the CGHs on the CRs. These CGHs are designed in such a way that, when used together with the M4 CR, they act like lenses that focus a collimated beam onto a common plane near the bottom of the WFC. When the M4 CR is used alone, it also focuses the collimated beam onto the same plane and therefore this spot can serve as the reference. The centroid offset of the focus on the common plane strongly depends on the centration of a mirror (M2, M3, or M5) relative to M4. This property is much less sensitive to tip/tilt errors of the mirror and thus can serve as a direct way to determine the decenter error of the mirrors relative to M4. When the CRs are used independently under the collimated beam, the CGH surfaces reflect some fraction of the beam back. This reflection can be used to constrain the tip/tilt of the mirrors relative to M4. The final mirror separations are constrained again by using the CR SMRs and LT and this process iterates until all DOFs are fully constrained per specification. As the go/no-go test, the two pairs (M2-M3, M4-M5) can be tested separately using the two subsystem $\mathrm{CGH}$ tests and the conjugate test and the final system alignment is confirmed by conducting the full system CGH test. The optical layouts of these CGH tests are shown in Figure 11.

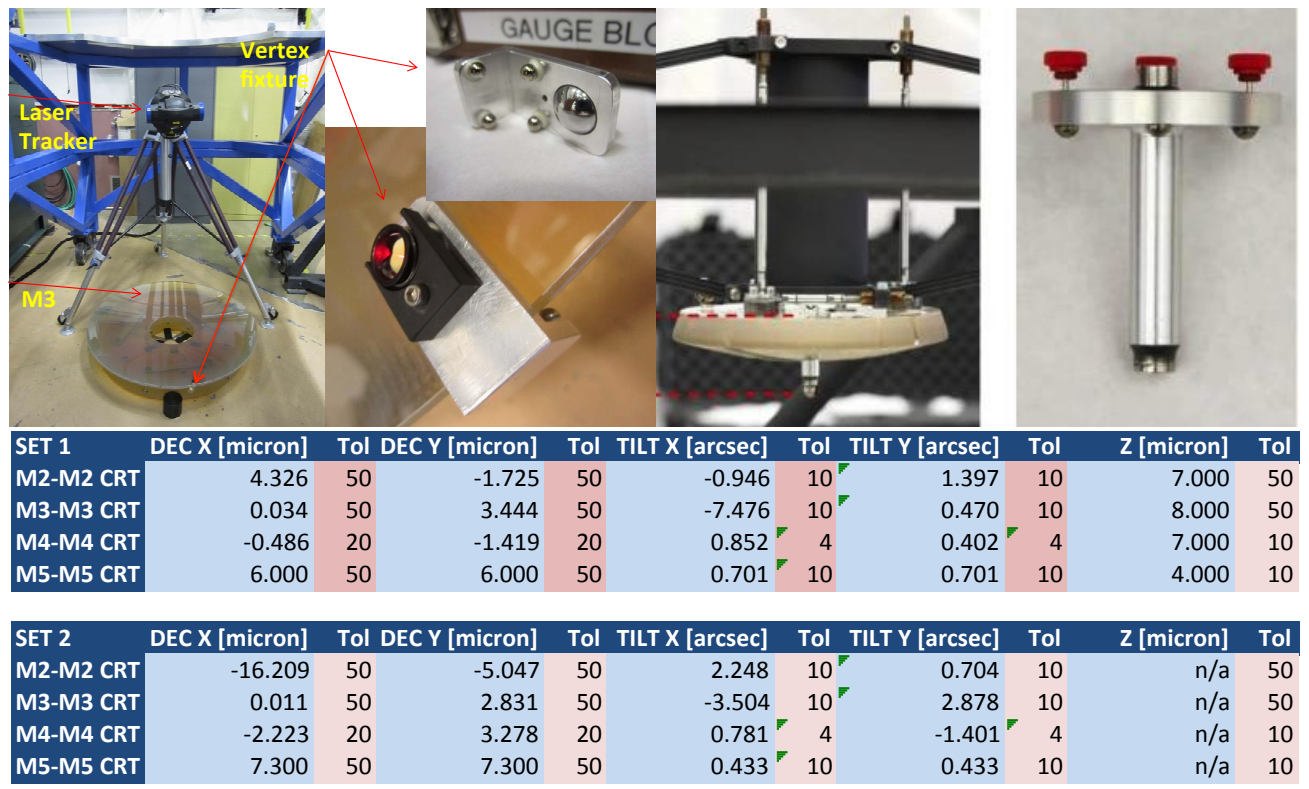

Figure 10 Vertex registration and final registration quality.
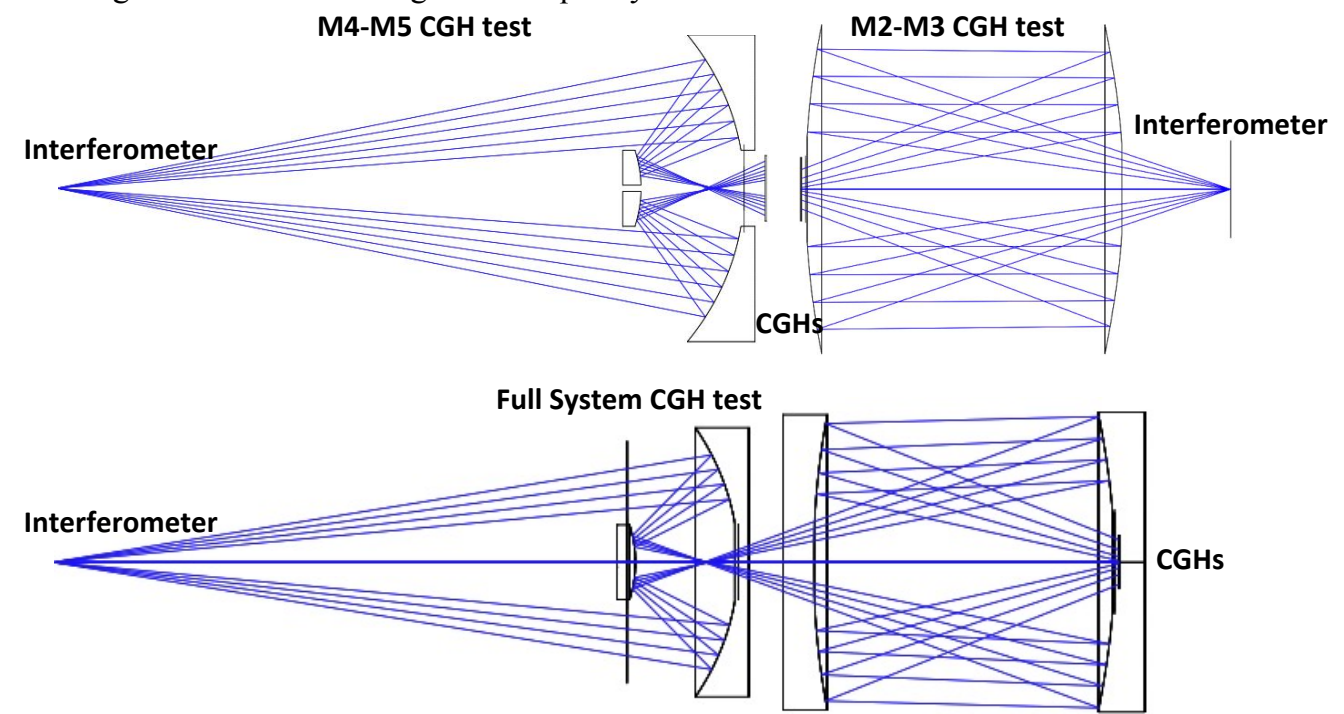

Figure 11 The optical layouts of the subsystem CGH tests and the full system CGH test. 
In summer 2013, however, we discovered that many of the CRs substantially drifted from their original locations and therefore their registration to the mirrors had been affected. This triggered a set of investigations to understand what happened to these CRs and to what degree the registration was affected. Although we had additional markers and small mirrors glued to each CRs for the purpose of capturing the original locations of the CRs, it was later found out that these features did not completely clarify the amount of drift, particularly in tip/tilt DOFs. Each mirror had two sets of CRs and the comparison between the sets gave us the relative drifts, which were $2 \sim 3$ times larger than the required tip/tilt tolerances, i.e. the CRs lost their tip/tilt registration. The main culprit of the drift turned out that, for some reason, RTV, instead of environmentally stable UV curing epoxy, was used to bond the CRs to their cell structures after the registration. The RTV is well known for its susceptibility to environmental changes, especially temperature and humidity, and thus usually used where dimensional stability is less critical. Also, the actual curing time of RTV appeared to be much longer than advertised. The application of the RTV to the CRs (in terms of the locations, thicknesses, and volumes around the CRs that were bonded with RTV) was also inconsistent across the CRs. This was a complete blow to the entire alignment plan that has been developed over the prior years and became a threat to the entire project.
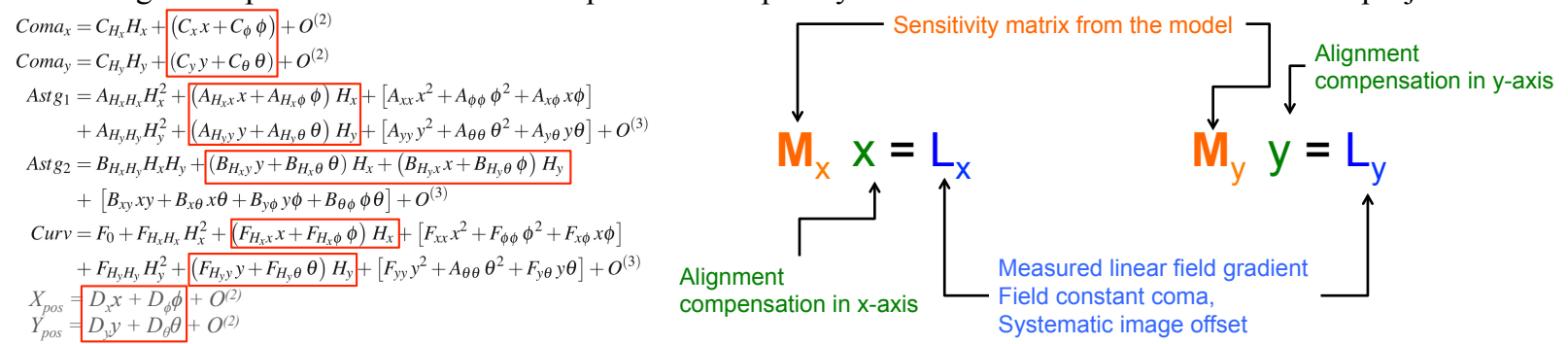

Figure 12 (Left) Core equations of the field aberrations, (Right) Principle of solving for alignment parameters.

Luckily, not all alignment registrations were lost. The M2 and M3 CRs' centration and height registration and thus those of SMRs appeared to be much less affected by the drift when compared to the alignment tolerances. For M5 CRs, it appeared that both tip/tilt and centration registrations were affected to an unusable level. For the M4 CRs, it was critical at least to estimate the absolute tip/tilt drift because this CR was the reference for all DOF in the entire system. This led to a separate test to quantify the absolute tip/tilt of the M4 CRs with respect to the M4 surface, which later confirmed that the M4 CRs appeared to be still intact in their registration to M4 in all DOF. This test was different from the original thorough test using interferometer and had a lot more simplified measurement scheme that did not fully sample the mirror surface. Therefore, there was some uncertainty associated with the state of the M4 CRs. However, having this test results, we proceeded with the alignment on the assumption that the other CRs were still usable at least to the extent of guiding the tip/tilt alignment to ballpark values as well as constraining the other DOFs of the mirrors. This reduced the problem down to a matter of making a new alignment plan that can constrain the tip/tilt alignment of the mirrors without using the CRs. At the time, we did not have time and resources to spend on repeating the registration processes for the mirrors, which would take at least 6 months to 1 year, and this was even more aggravated by the fact that we had to recoat M4 after its coating failure. Instead, we decided to adopt a plan to use the M2-M3 and M4-M5 subsystem CGH tests for the tip/tilt alignment. The new plan is as follows. In each subsystem test, align all mirrors in $\mathrm{x}$, $\mathrm{y}, \mathrm{z}$ DOF using the CRs and use tip/tilt of M2 and M3 in the case of the M2-M3 CGH test or tip/tilt and decenter of M5 in the case of the M4-M5 CGH test in order to null out the tip/tilt and coma aberrations. This plan was simple in theory, but in reality it was far more involved in terms of following the exact sequences of using different instruments for the purposes of measuring different quantities (e.g. Interferogram, relayed spots through the CGHs in the CRs, and LT position) and mounting/dismounting different fixtures for those measurements. In addition, the entire structure exhibited enough variation over time (i.e. temperature) to cause uncertainties in the measurements and this required painful iterations of measurements, adjustments, analyses, and verifications. The main disadvantage of this new plan was that it used up two independent subsystem CGH tests for alignment rather verification. Therefore, we essentially had only one verification test, which was the full system CGH test. Although the new plan could constrain the alignment of individual mirrors, the fact that the system CGH test only works on-axis made it difficult to gauge the off-axis performance of the system without an independent verification that can at least bracket the off-axis image quality.

Early on in this project, we developed a test scheme that became the M4-M5 conjugate test (Figure 5). As discussed in the early part of this section, the M4-M5 conjugate test utilizes the fact that this pair has two conjugate planes where stigmatic image can form within a $4 \mathrm{~mm}$ by $4 \mathrm{~mm}$ rectangular patches in $\mathrm{f} / 2$ beam. These are small patches but were enough in such a fast beam to produce field-dependent aberration signals that can be detected and interpreted in terms of the alignment state of this pair, which was very important since this pair essentially determines the off-axis performance 


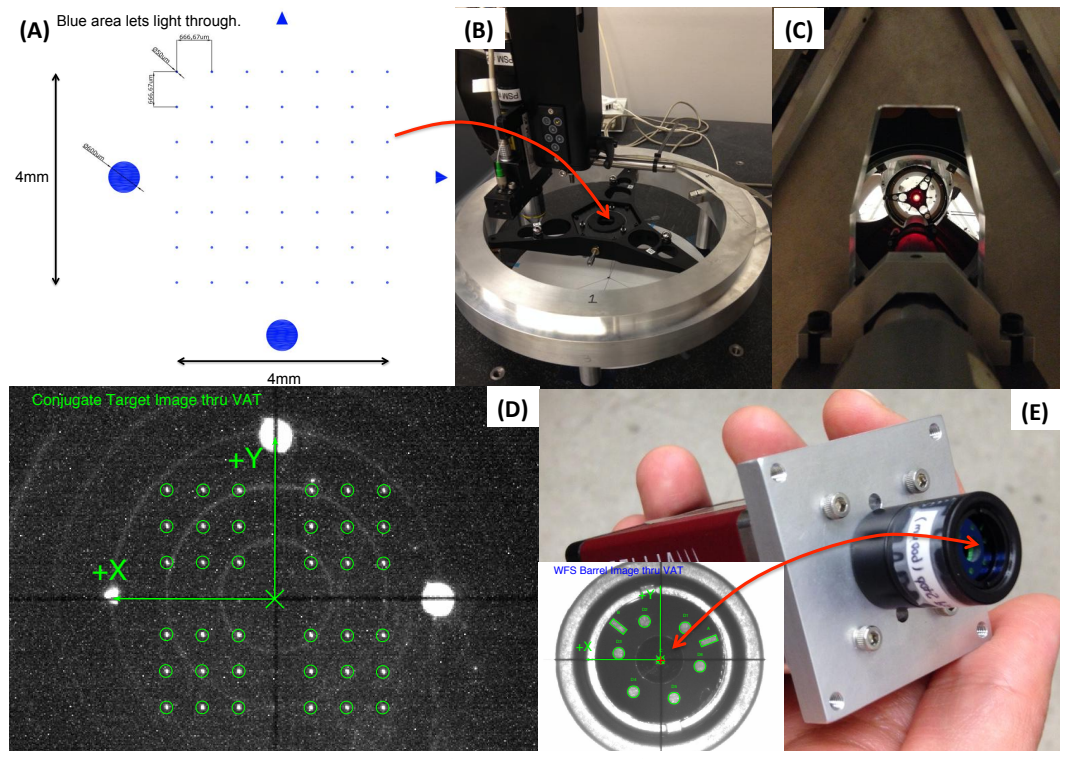

Figure 13 (A) Precision grid mask for the conjugate target, (B) the conjugate target's alignment to M4 CRs being transferred to the SMRs, (C) the conjugate target installed on M2 V-block mounts within the M2 central opening for the test and a collimated red LED illuminating the target, (D) Target image viewed through the video alignment telescope (VAT) that was used for the alignment of the target to the M4 CRs, (E) ShackHartmann wavefront sensor used in the measurement with its barrel mask imaged through the VAT for locating the center of the sensor. of the entire system[8]. We adopted this test as the additional verification test of the M4-M5 subsystem. The left panel of Figure 12 highlights the target aberrations in the conjugate test and the key terms in each aberration that are related to the alignment parameters of the M4 and M5. The right panel shows the basic principle of solving these equations for estimating the alignment parameters of M4 and M5 in this case. Note that this process focuses on the decenter and tip/tilt parameters and assumes that the separations are well constrained, which was the case for this test.

Figure 13 highlights some of the features of the conjugate target and the Shack-Hartmann wavefront sensors. The target consists of a precision grid mask (A) on a fused silica glass with almost no wedge between two surfaces. The target is housed in a cell with 5 axis adjusters builtin that facilitate the alignment of the target relative to the M4 CRs in-situ. This alignment is then later transferred to the SMRs glued on the conjugate target cell frame structure $(B, D)$, just like the mirror CRs to facilitate locating its $3 \mathrm{D}$ position within the LT measurement space. The target is installed on the same V-blocks in M2 as the M2 CRs are installed and illuminated by a collimated red LED from below (C). An engineered diffuser is used to ensure correct beam focal ratio emerging from individual pinholes within the grid mask. The M4-M5 pair relays these pinholes onto the plane where the wavefront sensor (E) on its own 5-axis piezo-motorized stage measures the wavefront at each pinhole, thus the fielddependence of the aberration result.

In early 2015, we executed the M4-M5 conjugate test and obtained the field-aberration data as shown in Figure 14. The data clearly indicated gradient present in the focus and two astigmatism modes, but much stronger in the astigmatism modes. There was also field constant offset in both coma modes. All these pointed to potential misalignment of the M4-M5 system. Because of the residual alignment errors of the conjugate target relative to the system reference (i.e. M4 CRs), however, we had to apply calibration offset to these measurements as shown in Table 2. The result was not exactly what we expected. As shown in its functional form in Figure 12, the gradient in the 45-deg astigmatism in one axis must normally shows up with the same magnitude but opposite sign in the 90-deg astigmatism in the other axis. The measurement did not exactly reflect this theory[9][10][11][12], which was an issue in translating the result into the alignment estimate of the M4 and M5.
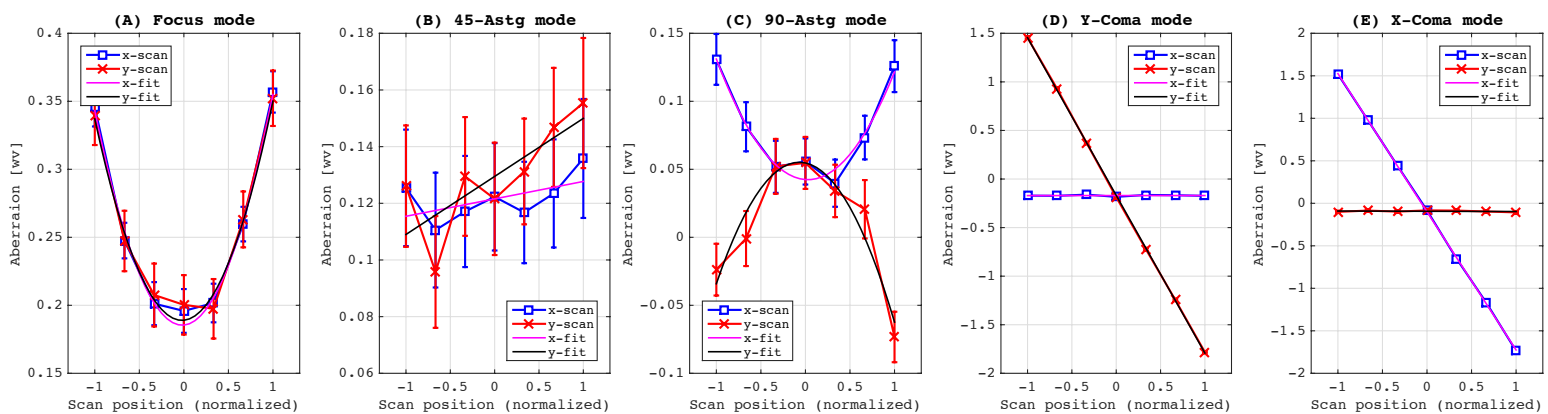

Figure 14 Five alignment-critical aberrations across the (normalized) field positions. 
Table 2 Summary of the detected gradients and offsets in five alignment-critical aberrations and their calibration.

\begin{tabular}{|c|c|c|c|c|c|}
\hline \multirow{2}{*}{ Terms } & \multicolumn{2}{|c|}{ Raw measurement } & \multicolumn{2}{|c|}{$\begin{array}{l}\text { Calibration offset due to } \\
\text { coordinate offsets }\end{array}$} & \multirow{2}{*}{ Unit } \\
\hline & $\begin{array}{c}X \text {-axis } \\
(2-\text { sigma })\end{array}$ & $\begin{array}{l}\text { Y-axis } \\
\text { (2-sigma) }\end{array}$ & $\begin{array}{c}X \text {-axis } \\
\text { (2-sigma) }\end{array}$ & $\begin{array}{l}\text { Y-axis } \\
\text { (2-sigma) }\end{array}$ & \\
\hline Linear field curv. & $\begin{array}{c}0.006 \\
(+/-0.002)\end{array}$ & $0.007(+/-0.004)$ & $\begin{array}{c}-0.0029 \\
(+/-0.0004)\end{array}$ & $\begin{array}{c}-0.0012 \\
(+/-0.0004)\end{array}$ & wV \\
\hline Linear field astg. 90 & $\begin{array}{c}-0.005 \\
(+/-0.002)\end{array}$ & $-0.014(+/-0.002)$ & $\begin{array}{c}-0.0009 \\
(+/-0.0001)\end{array}$ & $\begin{array}{c}0.0003 \\
(+/-0.0001)\end{array}$ & wV \\
\hline Linear field Astg. 45 & $\begin{array}{c}0.006 \\
(+/-0.002)\end{array}$ & $0.020(+-0.002)$ & $\begin{array}{c}-0.0009 \\
(+/-0.0001)\end{array}$ & $\begin{array}{c}-0.0003 \\
(+/-0.0001)\end{array}$ & wV \\
\hline $\begin{array}{l}\text { Field constant } \\
\text { coma }\end{array}$ & $\begin{array}{l}-0.100 \\
(+/-0.001)\end{array}$ & $-0.168(+/-0.001)$ & $\begin{array}{c}0.0202 \\
(+/-0.0033)\end{array}$ & $\begin{array}{c}0.0064 \\
(+/-0.0033)\end{array}$ & wV \\
\hline Image offset & $\begin{array}{c}-0.021 \\
(+/-0.035)\end{array}$ & $\begin{array}{c}-0.051 \\
(+/-0.035)\end{array}$ & $\begin{array}{c}0.023 \\
(+/-0.0038)\end{array}$ & $\begin{array}{c}0.0073 \\
(+/-0.0038)\end{array}$ & $\mathrm{mm}$ \\
\hline
\end{tabular}

However, this gave us a chance to explore various cases possible given the data. We analyzed the data with many different combinations of the gradient terms. A singular value analysis of the sensitivity matrices indicated that the least sensitive alignment mode (a combination of M4 and M5 decenters) was highly correlated with the gradient in the focus mode, thus a small variation in the focus gradient resulted in rather unrealistic large decenter errors in M4 and M5. Thus including and excluding this weakest mode in the analysis resulted in another set of possible misalignment cases. Finally, we also had the estimate from the previous alignment process, namely the LT data and the CR CGH data. Therefore, we estimated the alignment parameters under the constraint of these existing data. This analysis process resulted in twelve potential alignment cases for the M4-M5 pair. We modeled these possible M4-M5 alignment cases in the full system model in order to understand the ramification of these misalignment possibilities in terms of the full telescope performance. One out of these indicated strongly misaligned M4-M5 thus much degraded off-axis performance of the system. For the rest of the cases, the M4-M5 misalignment was small good enough not to degrade the system's off-axis performance, even though the misalignment itself was outside the required specification, thus successfully bracketing the degree of the M4-M5 misalignment. Based on the results from all these meticulously executed alignment tests, the system was regarded more than likely to perform at the level required by the overall science requirements, but the possibility of the worst case still remained. With this uncertainty in our mind, we moved onto the final phase of the alignment process.

The full system CGH test is another independent test that can verify the alignment of the system in a go/no-go fashion. This is an on-axis test and as such off-axis information is obtained. However, we had a set of expected probability functions that each of the alignment critical aberration must reproduce in order for the system to be declared to have passed the test. Also, this test gave us a very good chance to examine whether our design and manufacturing of the extra aspheric corrector lens, that was based on the M3 and M5 prescription estimates, was successful or not. For that, we also had specific probability functions of each prescription-critical aberration as the pass/fail criterion. In addition, this test gave us an opportunity to verify the structural soundness by testing it at $35 \mathrm{deg}$ angle, which is the nominal angle that the WFC would operate at the top of the HET. We already tested the structure with dummy mirrors in 2011 , but this was the opportunity to test it with the real aligned mirrors. The optical layout of the system CGH test is shown in Figure 11 and we summarize the outcome of the test below.

Figure 15 shows the probability distributions of the prescription-critical aberrations in the full system CGH test with the extra aspheric corrector lens. The red-color distributions correspond to the system with the adopted M3 and M5 prescriptions. Other colored distributions are based on other prescriptions estimated from subsequent subsystem tests. The black narrow distributions correspond to the measured aberrations. The results indicated that the measurement strongly favored the adopted M3 and M5 prescription against other potential possibilities. It also meant that the extra aspheric corrector lens that we designed and manufactured to compensate the excess spherical aberration was effectively doing its function. The $3^{\text {rd }}$ order spherical aberration of the system is now reduced to 0.2 waves from more than 1 waves. In terms of the alignment, we measured the positions of the system $\mathrm{CGH}$ and the interferometer relative to the system reference, i.e. M4 CR and this confirmed the correct placement of these test components. Note that the CGH was used as a compensator. This compensation has the same effect as the rigid body motion of the WFC relative to the HET M1 and this is exactly how we operate at the telescope for compensation. For the given alignment tolerances, the CGH compensation had specific probability distributions and the measured CGH position must be within those distributions. 
The measured CGH positions were well within the required distributions. Subsequently, we examined the alignmentcritical aberrations (Figure 16), which were in good agreement with the expectations.
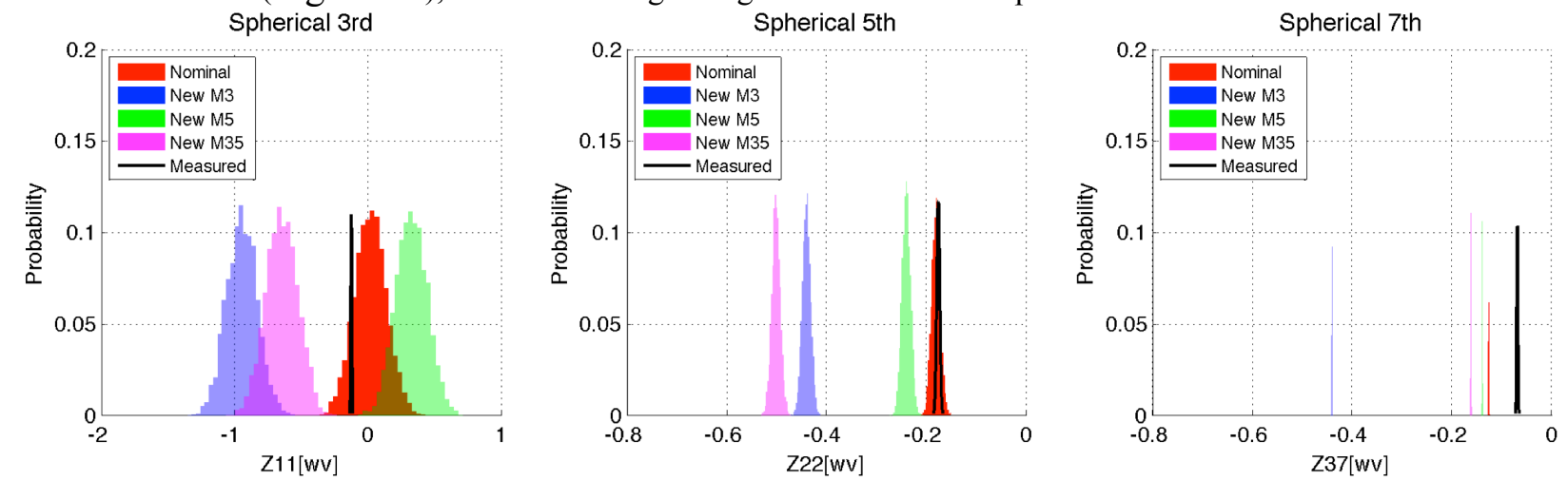

Figure 15 The probability distributions of the prescription-critical aberrations compared to the measurements. Nominal means the M3 \& M5 prescriptions we adopted and New M3, M5, M35 are variations estimated from the subsequent subsystem CGH tests.
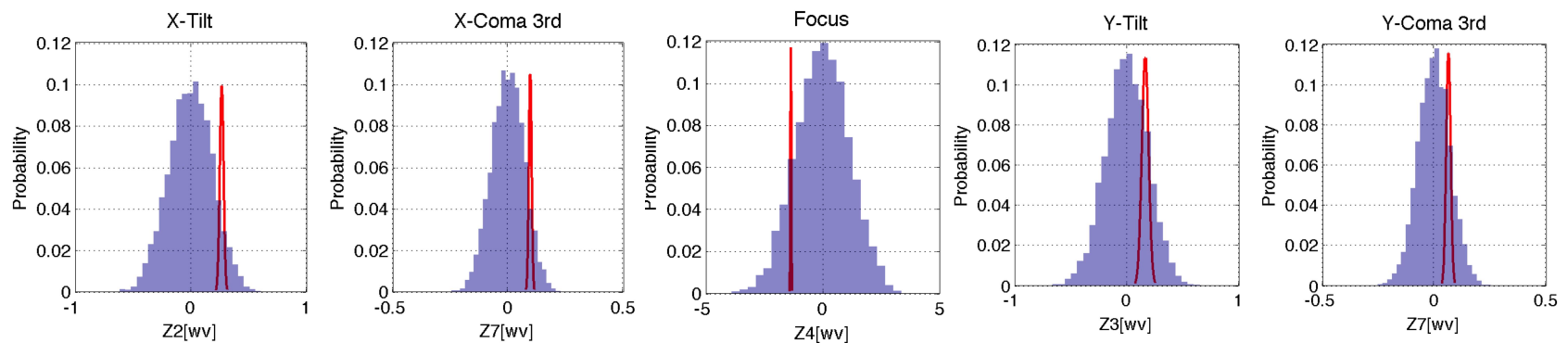

Figure 16 The probability distributions (Blue) of the alignment-critical aberrations and their measured distributions (Red).

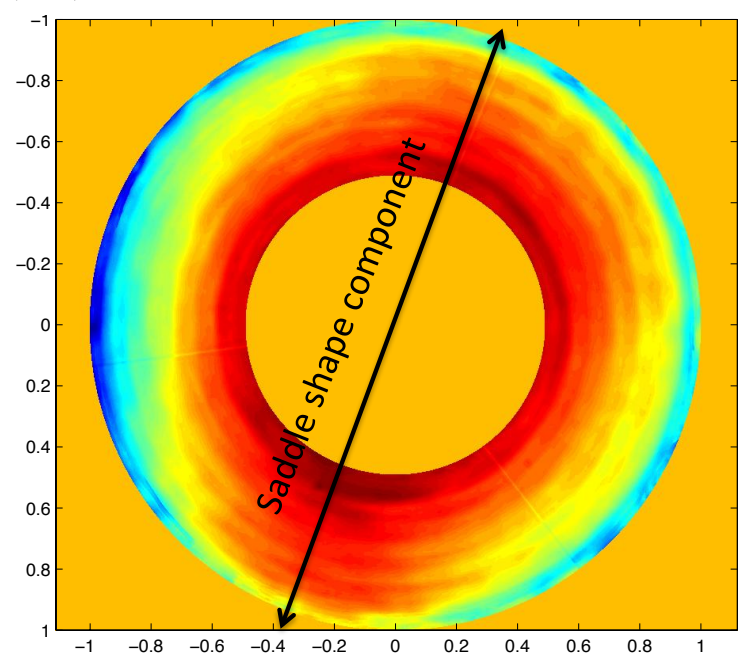

Figure 17 A wavefront map of the full system with the aspheric corrector lens.
The analysis of the system CGH test result also revealed that the system exhibited 0.5 waves of astigmatism and 0.27 waves of trefoil aberrations (Figure 17). These aberrations turned out to be coming from the aspheric corrector lens. The aspheric corrector lens is a $280 \mathrm{~mm}$ diameter and $10 \mathrm{~mm}$ thick fused silica glass and its high aspect ratio under gravity and mounting stress could have cause it. Through out the repeated system CGH test with/without the aspheric corrector lens, we confirmed that this effect was stationary without any variation. We thus modeled this effect in the full system design and confirmed its contribution to the overall telescope imaging performance to be insignificant. This is largely because the telescope system is single-path and thus the amount of aberrations seen in the system test (in double-path) is halved. We continued with the system test at the nominal $35 \mathrm{deg}$ angle and the variation in the measurement was within the expected range and, when the system was brought back to the vertical orientation, all components went back to their original positions with small variations.

\section{TRANSPORTATION AND ON-SITE VERIFICATION}

On April 22 and 23 of 2015, we held an extensive external review to assess the status of the WFC system, to identify the potential risks in terms of performance as well as other important record-keeping and long term maintenance aspects of 
the system at the HET, and to evaluate our plans to further refine the understanding of the WFC image quality on the HET and to mitigate risk factors that may arise once the system is deployed at the HET. The on-sky verification of the WFC alignment within the full telescope system was the key aspect and we will discuss this in the next section. In this section, we highlight the transportation of the WFC system from Tucson, AZ to the HET in Fort Davis, TX and then the on-site ground verification that confirmed the safe transportation of the system.

For the transportation, we have designed and constructed a shipping container as shown in Figure 18. The total weight of the WFC system and its maintenance cart is approximately $4600 \mathrm{lbs}$. To minimize shock during the transportation, we used four 5/8-inch cable isolators with the compression spring rate of $1199 \mathrm{lb} / \mathrm{in}$, the compressive maximum deflection of $2.2 \mathrm{in}$, shear/roll spring rate of $641 \mathrm{lb} / \mathrm{in}$, and shear/roll maximum deflection of 2.2in. These isolators provided isolation protection in all axes. The isolators were mounted to the shipping base weldment made out of mild steel 4inx8in rectangular tubings. A thin (22 Gauge sheet) aluminum weldment floor was then attached to the base weldment. Four casters were then used underneath the base weldment in order to position the entire shipping container inside the HET receiving bay. To environmentally protect the system from outside, we also made the shipping box top that consists of seven panels of 22 Gauge sheet aluminum weldment. This box was attached to the shipping base once the WFC in its maintenance cart were loaded and secured to the cable isolators. To add a secondary protection of the system from moisture in particular, we wrapped the entire WFC with two cylindrical moisture bags that were sealed from outside with dry nitrogen filled and humidity monitoring stickers inside. For loading and unloading, we built swivel eye bolts (rated to 17,630 lb for vertical lift and 7,050 lb for angular lift load) at the four corners in the shipping base weldment that were hooked to a 5-ton capacity load spreader above the top of the shipping container through 12-ft straps. A crane was then used to pick up the load spreader from the top to lift the entire shipping assembly. We used a Hoyt UltraShock sensor to record temperature, humidity, pressure, and 3-axis acceleration. In addition, we installed single-use analogue shock indicators in various places around the WFC system as a back up[13].

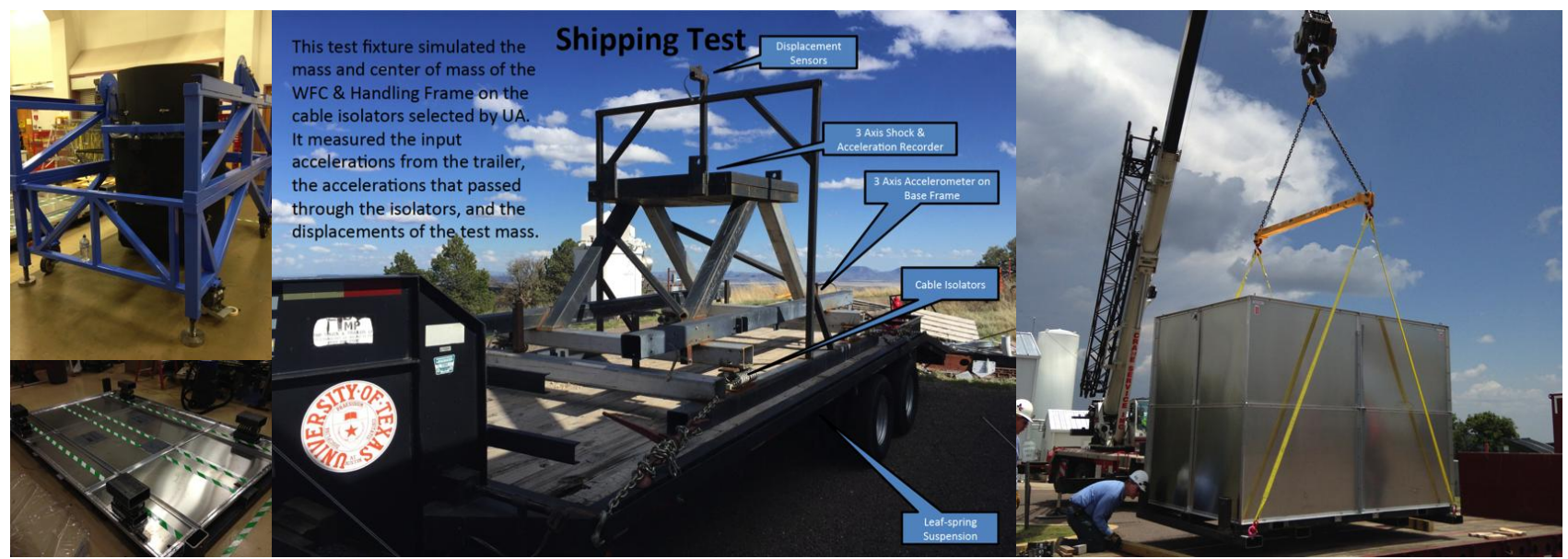

Figure 18 The shipping container of the WFC system. (Top left) the complete WFC system at the transportation orientation within its maintenance cart. (Bottom left) the shipping base with cable shock isolators. (Middle) The mockup shipping test to verify the cable isolator performance. (Right) the delivered shipping container with the WFC inside at the HET parking lot with a crane lifting the assembly to load into the HET receiving bay.
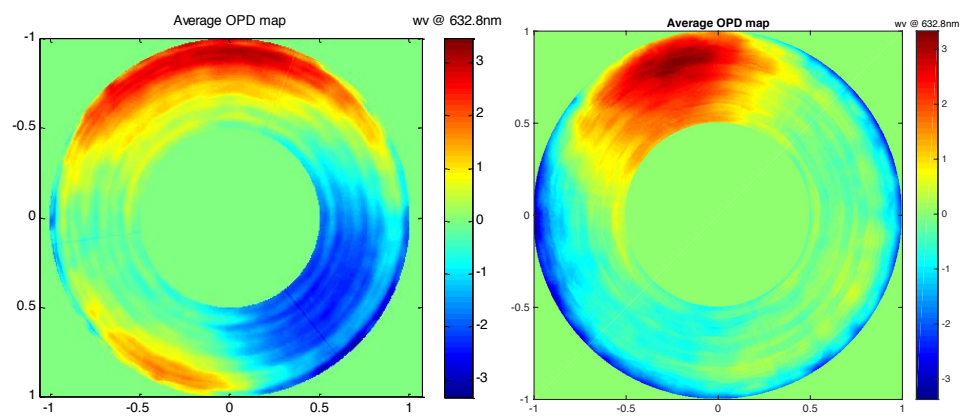

Figure 19 A side-by-side comparison of the system CGH test wavefront maps measured $(0.753 \mathrm{wv} \mathrm{rms})$ at Tucson (Left) and $(0.767 \mathrm{wv} \mathrm{rms})$ at HET (Right).
Before the design of the shipping container, we estimated the shock limits via the Finite Element Analysis (FEA) models of the system. The model was analyzed in terms of the structural displacements and the margins of safety for forces applied to the axial and tangent mirror support rods and the stresses applied to the bond lines that interface the mirrors and those support rods. Through these analyses, the maximum level of accelerations at which the system still maintains the alignment was determined to be $3 \mathrm{~g}$ in the lateral directions and $5 \mathrm{~g}$ in the vertical direction. In order to ensure that the chosen cable 
shock isolators are up to the task and to assess the deflections, we conducted a mock-up transportation experiment, which resulted in the expected WFC accelerations less than $0.55 \mathrm{~g}$ in lateral directions and $0.7 \mathrm{~g}$ in the vertical direction with the deflections less than $1 / 2$ of the clearance of the WFC within the shipping container. This test also resulted in a transportation protocol that the maximum speed of the transportation vehicle must be slower than $45 \mathrm{mph}$ to minimize any potential shock due to rough road conditions on the interstate highways and local roads.

Table 3 The on-site verification matrix.

\begin{tabular}{|c|c|c|c|}
\hline \multicolumn{2}{|c|}{ Verification item } & Requirement & PASS / ACCEPTABLE / \\
\hline \multicolumn{2}{|c|}{ Shock value } & $<3 \mathrm{~g}$ lateral, $<5 \mathrm{~g}$ vertical & PASS (0.8 max.) \\
\hline \multicolumn{2}{|c|}{ Humidity } & $<100 \%$ (condensing) & PASS (12.7 61.1\%) \\
\hline \multicolumn{2}{|c|}{ Temperature } & $-25 \operatorname{deg} C \sim 66 \operatorname{deg} C$ & PASS (17.8 27.6deg C) \\
\hline \multicolumn{2}{|c|}{ Visual inspection } & Damage inspection & PASS (no damage found) \\
\hline \multirow{6}{*}{$\begin{array}{l}\text { Syste } \\
\text { m } \\
\text { CGH } \\
\text { test }\end{array}$} & \multirow{3}{*}{ Compensable aberrations } & Tilt $<1 \mathrm{wv}$ & PASS (0.73 wv max.) \\
\hline & & Coma $<0.25 w v$ & PASS (0.114 wv max.) \\
\hline & & Focus $<4 w v$ & PASS (0.289 wv max.) \\
\hline & Non compensable aberrations & Magnitude change less than $0.05 \mathrm{wv}$ & $\begin{array}{l}\text { ACCEPTABLE (main change by } 0.085 \mathrm{wv} \text { in spherical } \\
\text { aberrations in single path } \rightarrow \text { model expects the HET } \\
\text { to maintain its performance as required). }\end{array}$ \\
\hline & Interferometer focus position & Magnitude change $<50$ um & PASS \\
\hline & Compensated CGH position / angle & $\mathrm{X}, \mathrm{Y}, \mathrm{Z}<150 \mathrm{um}, ; \mathrm{Rx}, \mathrm{Ry}<125$ urad & PASS \\
\hline
\end{tabular}

On May 27 of 2015, the WFC was loaded onto an air-ride truck in Tucson. The loading occurred in the early evening and the transportation happened overnight to avoid heat since the shipping container was not air-conditioned. The transportation took about 14 hours at $45 \mathrm{mph}$ speed on the interstate 10 and much slower on the local road leading up to the HET around Fort Davis. The arrival and unloading of the WFC took place on May 28 and was smooth. The system was unpacked in the subsequent days inside a clean tent set up in the HET receiving bay. The tent was sealed from outside, temperature and humidity controlled, and over-pressurized to minimize dust, mouse, and moth ingress. We visually inspected the mirror coating, mount joints, epoxy lines, flexures, and supporting rods to identify any damages on the system. Also the environmental data from the UltraShock sensor was downloaded and reviewed. We then conducted the system CGH test as the last step of the on-site verification. All visual inspection and shock record indicated the safe delivery of the WFC system and this CGH test was the final confirmation. The same test done in Tucson before the shipping could be directly compared to the result from this test as shown in Figure 19 and in Table 3. The result confirmed the successful transportation of the system.

\section{END-TO-END TELESCOPE ALIGNMENT AND ON-SKY VERIFICATION}

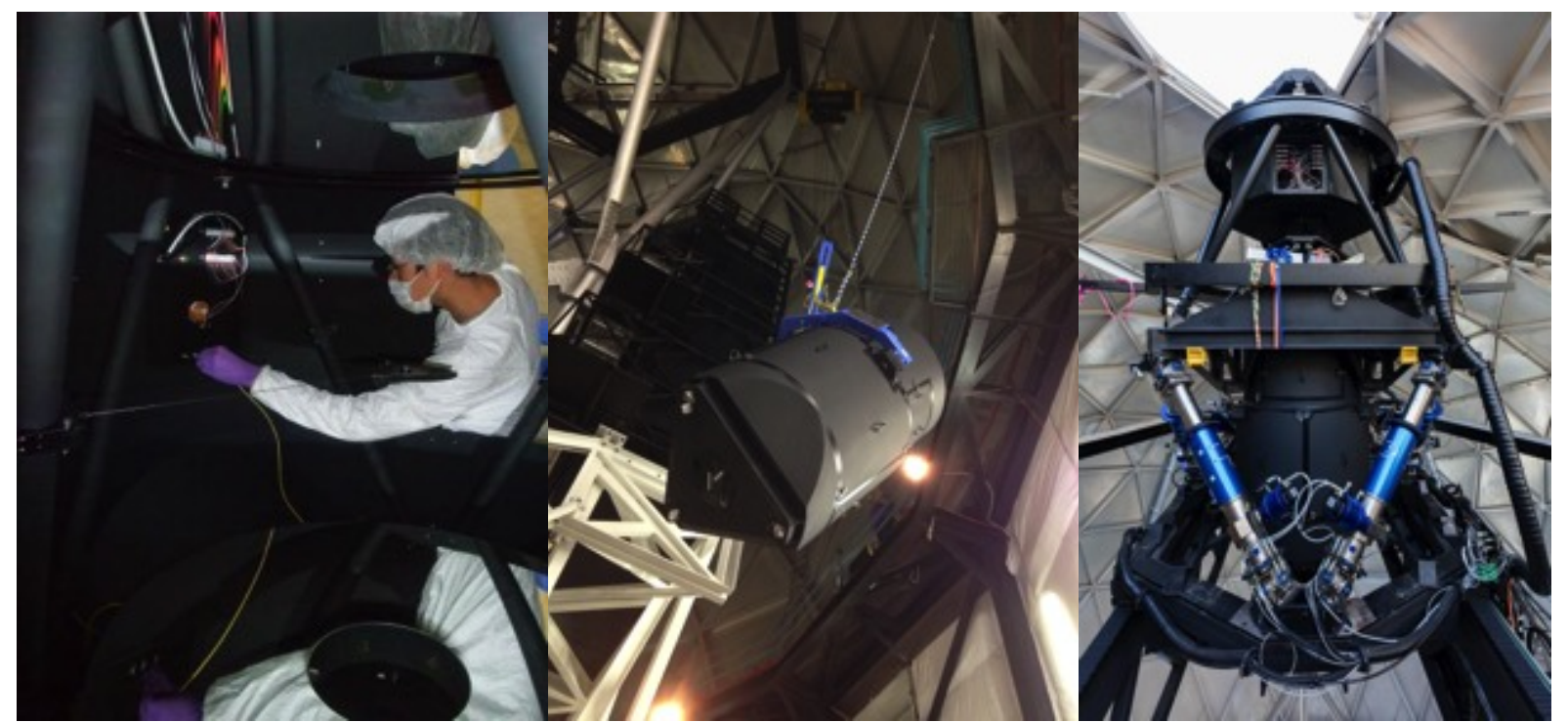

Figure 20 (Far left) Installing DMI fibers to the WFC instrument panel, (Left) WFC being lifted, (Right) WFC and the Prime Focus Instrument Package (PFIP) assembled on the HET tracker, (Far right) WFC + PFIP solid model. 


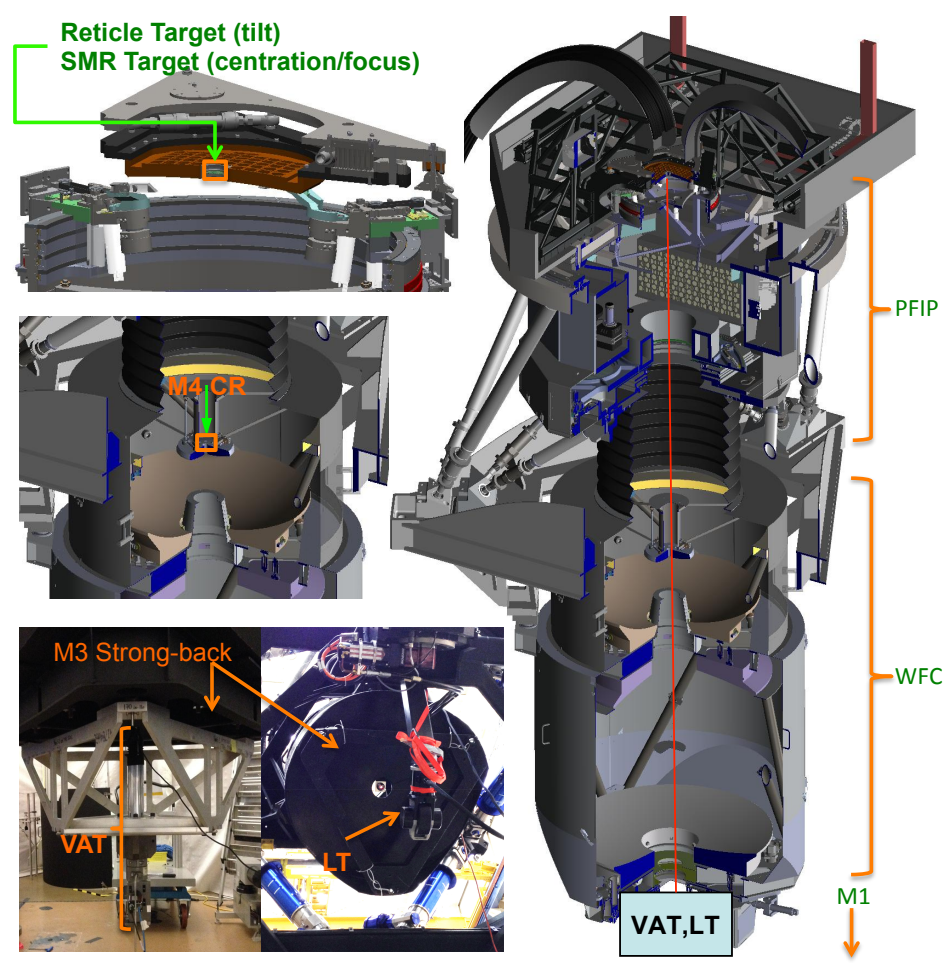

Figure 21 Layout of the PFIP/WFC/M1 and their references and instruments used for the end-to-end telescope alignment.
After the on-site verification, we modified some of the outer skin weldment to ensure better fit and seal of the WFC for effective nitrogen purge to over-pressurize the system. This resulted in the expected nitrogen flow rate. We also added several parts to the system for later mounting the distance-measuring interferometers (DMI) and the low instrument package (LIP). On July 2 of 2015 , the WFC was lifted and safely installed on the HET (Figure 20). Once the WFC was installed, the Prime Focus Instrument Package (PFIP) was mounted. The PFIP contains the focal plane assembly (FPA) where various telescope guiding/metrology systems are operating including an acquisition camera, two guiders, two low-order operational wavefront sensors, one high-order calibration wavefront sensor, and a pupil-viewing camera[14][15]. Also, the FPA contains the input head mounting plate (IHMP) where all of the VIRUS IFUs and other facility instruments' fiber cables are mounted. In the Austin lab, all these PFIP instruments were aligned to the IHMP using the combination of a video alignment telescope (VAT) and a laser tracker (LT) to the required accuracy. On the telescope, we needed to align the PFIP, the WFC, and the M1 with respect to each other (Figure 21). This end-to-end telescope alignment was done using the VAT and the LT mounted around the bottom of the WFC. We used the M4 CR as the system reference and aligned the PFIP to the M4 CR in all DOFs. For the alignment of the WFCPFIP to M1, we bonded a $1 / 2$-inch SMR on the center of the center segment of M1 for the LT focus measurement and used the direct reflection from the same segment and the image of the SMR for the VAT translation and tip/tilt measurements. By these means, we achieved the full telescope alignment better than the specifications in all DOFs (Table 4). With these final telescope alignment results, we entered into the on-sky WFC alignment and HET imaging performance verification stage.

Table 4 The end-to-end PFIP/WFC/M1 alignment results.

\begin{tabular}{|l|r|r|r|}
\hline \multicolumn{1}{|c|}{ Critical alignment } & Centering (spec) [mm] & Focus (spec) [mm] & Tip/tilt (spec) [asec] \\
\hline \hline WFC-PFIP & $0.059(0.170)$ & $0.006(0.300)$ & $6(90)$ \\
\hline M1-WFC & $0.045(0.100)$ & $0.010(0.015)$ & $6(15)$ \\
\hline
\end{tabular}

The primary goal of the on-sky verification is to evaluate the alignment status of the WFC within the full telescope system. Unlike other WFC-only lab tests, this allows us to directly assess the full telescope field of view and measure the complete off-axis imaging performance. The field dependence of the telescope image quality can then be interpreted in terms of the alignment status of the WFC and also be used to evaluate the telescope imaging performance. In order to execute this measurement, we designed a multiple wavefront-sensing test as follows. The test consists of 9 ShackHartmann wavefront sensors distributed along two orthogonal axes over the field of view, thus 5 sensors in each axis. This allows super-critical sampling of the five major field aberrations (i.e. the field curvature, two astigmatisms, and two coma) to exactly determine their overall offset, linear gradient, and parabolic field dependency as shown in Figure 12.

We built $9 \mathrm{SH}$ wavefront sensors with 20 sub-apertures across the pupil diameter. These sensors are called the Deployable Wavefront Sensor (DWFS). The sensor grid geometry is hexagonal with roughly 300 sub-apertures over the annular telescope pupil with about $16 \%$ central obscuration in area. Each sensor consists of the standard wavefront sensor body and the field stop fixture. The main body is then comprised of a collimation doublet and a hexagonal micro lens array and a CMOS detector. The field stop fixture consists of a pinhole mask glued to an aluminum block that rides on mini 3-axis adjusters with three clamping screws. Each sensor has two different field stop fixtures. The pinhole in one of them matches the field of view of each sub-aperture and this stop fixture is used in measuring the telescope 
obscuration under twilight illumination and in the normal measurement operation. The other stop fixture has a much smaller pinhole and is used to measure the calibration data of each sensor, which is then subtracted from the actual measurement. The wavefront sensor body was fabricated to fit into the IFU slots in the IHMP. Although these IFU slots were fabricated to accuracy better than $0.01 \mathrm{~mm}$ in all axes, individual wavefront sensors were not fabricated to the same accuracy. That was why we made the field stop fixtures adjustable, which allowed us, under a high magnification microscope, to accurately position and register the pinholes to a common $(x, y, z)$ position with respect to the same reference fixture so that the variation across the individual sensors can be later calibrated out. This reference fixture was very tightly toleranced to match that of the IHMP. Also the fixture had a $1 / 2$-inch SMR nest at its tip on which a high precision tooling ball can be placed. The center of this tooling ball matches the vertex of the IHMP surface when the reference fixture is installed in the center slot of the IHMP. Thus by aligning all field stop fixtures to this reference fixture in the lab all wavefront sensors are optically par-focal to each other and to the IHMP surface. This eliminated the systematic tilt in the wavefront sensor across the field, which would have manifested as an artificial focus gradient in the measurement. Finally, we designed the kinematic interface between the wavefront sensor body and the field stop fixture, i.e. mini v-blocks on the wavefront sensor body and mini spherical actuator tips on the field stop fixtures. This ensured repeatable mounting and dismounting of the field stop fixtures to/from the wavefront sensor body. We also embedded magnets in this kinematic interface as a way to secure the field stop fixture to the sensor body instead of using fasteners. This eliminated the tightening/untightening of screws and the associated shift of the field stops relative to their preregistered positions. By using these procedures, we were able to make all 9 DWFS par-focal to each other to the accuracy better than $0.025 \mathrm{~mm}$ in focus and $0.035 \mathrm{~mm}$ in translation, including the kinematic repeatability. This resulted in the overall alignment error of $(x, y)=0.081 \mathrm{~mm}, z=0.061 \mathrm{~mm}$, and tip/tilt $=66 \mathrm{asec}$ in setting up the WFC-FPA-DWFS on the telescope, compared to the specification of $(x, y)=0.170 \mathrm{~mm}, z=0.3 \mathrm{~mm}$, and tip/tilt $=90$ asec.
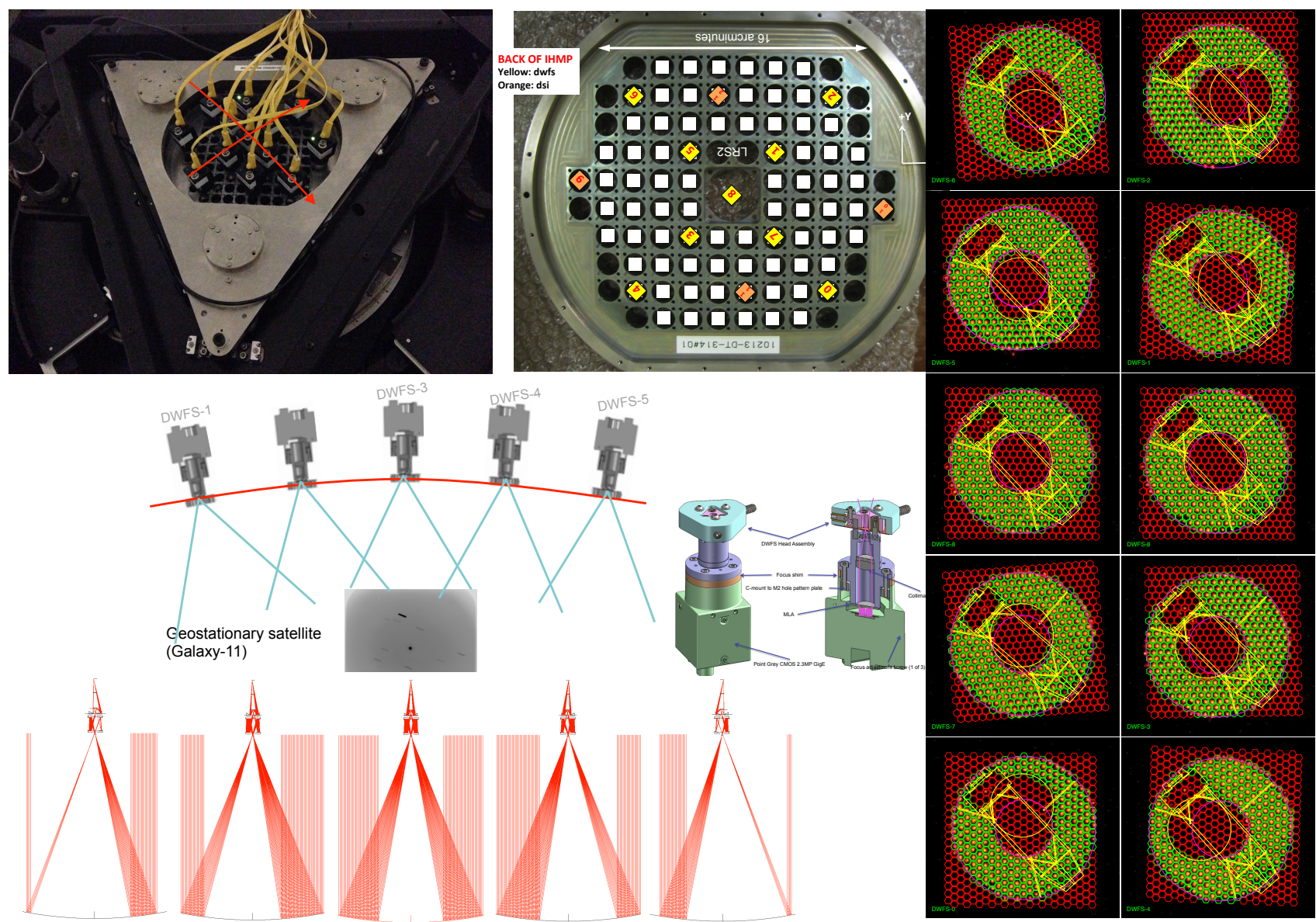

Figure 22 (Top left) 9 DWFS and 4 Imagers mounted in the IHMP, (Top middle) Locations of the DWFS in the IHMP, (Bottom left) Measurement sequence using a geo-stationary satellite and opto-mechanical view of the DWFS, (Right) Images from 9 DWFS on the telescope with SH grid and telescope geometries overlaid. 
Once the lab alignment and registration were complete, we installed the DWFS on the telescope. The calibration consisted of two steps. In the first, we placed the smaller pinhole field stop fixture and exposed the telescope and DWFS under bright twilight illumination. This resulted in the images of SH spots with centroid shifts solely due to the internal to individual sensors, which were used as the calibration data. In the second step, we then swapped the larger pinhole field stop fixtures in and made another set of twilight exposures. This resulted in the images that revealed the pupil boundaries and various telescope obscuration geometries seen from individual wavefront sensors. This formed the basis of the pupil obscuration masks that were later used to build the modal fitting matrices for individual sensors. With all of the calibration data in hand, we executed the test throughout September in 2015. For the test, we used some of the geostationary satellites (GOES-14, DIRECTV-10, GALAXY-7) instead of stars as the wavefront sensing target. The use of a satellite enabled the telescope to be parked near the center of the M1 and thus eliminated the dynamic hardware deflection and the errors associated with the telescope mount models while moving the tracker/WFC/PFIP to track a star. This also minimized the variation of the pupil illumination for all DWFS and reduced the uncertainties associated with it.

At the beginning of the test, we have not fully tested offsetting the telescope pointing to different parts of the focal surface. After a few nights of offset tests, we were able to hit individual DWFS centers quite efficiently with accuracy well within the field size of the sensors (5.7 arcsec in diameter). The test was conducted in the following fashion. We started each set of measurements by pointing a satellite to the DWFS in the field center and taking data from it. We sequentially pointed the satellite to the next DWFS and took the data. We then moved back to the center DWFS before going to the next DWFS and repeated this process until we took the data from the last DWFS. This resulted in the data set that consists of each off-axis DWFS data paired with the on-axis DWFS data. This enabled us to track the temperature variation of the telescope structure (mostly in the focus motion detected by the distance measuring interferometer (DMI) ) and the M1's global shape variation including its radius of curvature and later on to calibrate out the variation of these effects between different DWFS data. Since the telescope was very fresh and not all of the operation procedures/software had not been thoroughly tested at that point. This resulted in some data set being discarded due to faults in the telescope operation during the tests. Also the local weather played a substantial role in determining the size of useful data sets we obtained. Throughout the 4-week long wavefront sensing campaign, total 16 independent data sets were gathered.
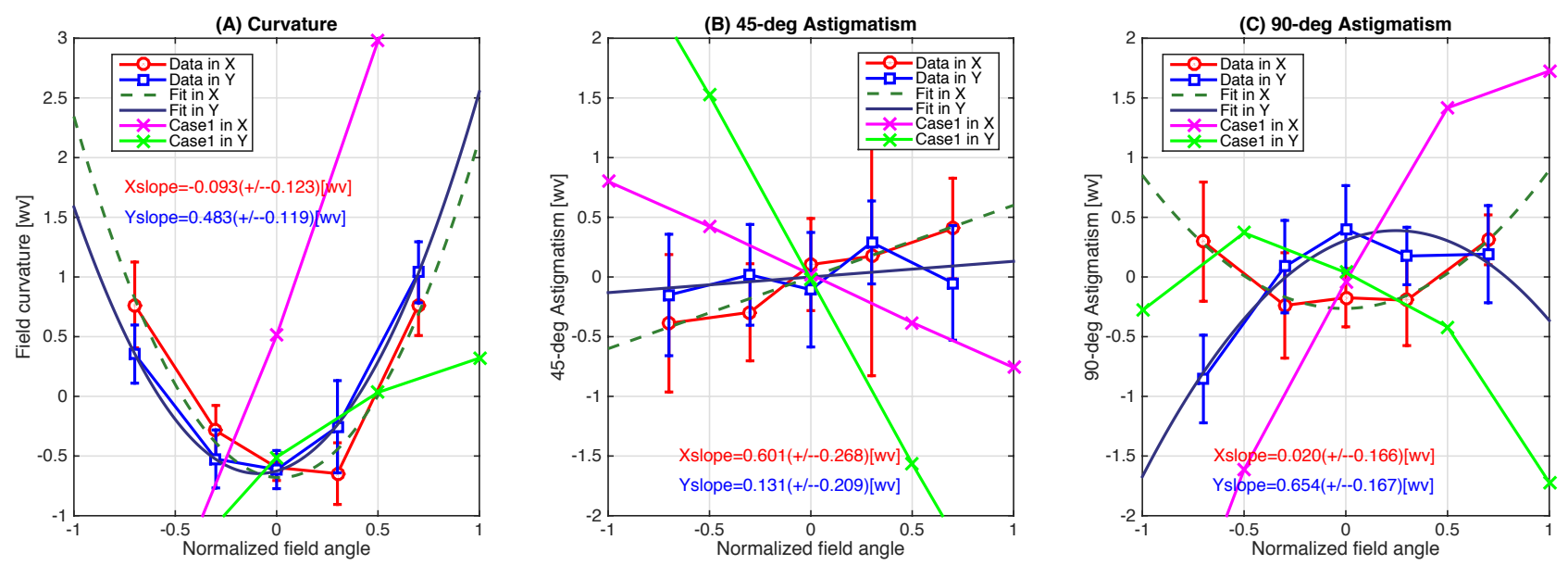

\begin{tabular}{|c|c|c|c|c|c|c|}
\hline \multirow{2}{*}{ Axis } & \multicolumn{2}{|c|}{ Curvature [wv] } & \multicolumn{2}{c|}{ 45-deg Astigmatism [wv] } & \multicolumn{2}{c|}{ 90-deg Astigmatism [wv] } \\
\cline { 2 - 7 } & $\begin{array}{c}\text { Data } \\
\text { (95\% limit) }\end{array}$ & Case \#1 & $\begin{array}{c}\text { Data } \\
\text { (95\% limit) }\end{array}$ & Case \#1 & $\begin{array}{c}\text { Data } \\
\text { (95\% limit) }\end{array}$ & Case \#1 \\
\hline X-axis & $0.483(+/-0.14)$ & 3.1 & $0.131(+/-0.29)$ & -0.7 & $0.654(+/-0.23)$ & 3.0 \\
\hline Y-axis & $0.093(+/-0.13)$ & 1.5 & $-0.601(+/-0.32)$ & -3.0 & $-0.02(+/-0.19)$ & 0.7 \\
\hline
\end{tabular}

Figure 23 (Top) The measured field curvature and two astigmatisms from the 9 DWFS, (Bottom) the summary of the field-linear gradient statistics and the comparison to the estimates by the worst WFC alignment case predicted by the M4-M5 Conjugate test.

Figure 23 shows the plots of the measured field curvature and two astigmatisms from the 9 DWFS. The green- and magenta-colored solid lines represent the field-dependence of these aberrations if the WFC alignment were similar to the worst case as predicted by the M4-M5 Conjugate test. The table at the bottom summarizes the field-linear gradient 
statistics of each aberration in each field axis. First of all, the results indicate that we detected these field-linear gradients well above the noise limit in the 16 independent data sets across one months of variation in the observing environment. Secondly, the gradient values in the 90-deg astigmatism showed up in the 45-deg astigmatism with the opposite signs, especially between the X-axis gradient of the 90-deg astigmatism and the Y-axis gradient of the 45-deg astigmatism. This is exactly what was predicted by the theory as discussed in Figure 12. The Y-axis gradient of the 90-deg astigmatism and the $\mathrm{X}$-axis gradient of the 45-deg astigmatism do have opposite signs, but their magnitudes do not match, but one should not that their detection uncertainties are much larger. Therefore, we had strong confidence in the detected gradient values in these data sets. Finally, the detected gradients strongly favored the much benign WFC alignment cases (which were favored in the M4-M5 conjugate test data as well as other alignment test results) against the worst case.

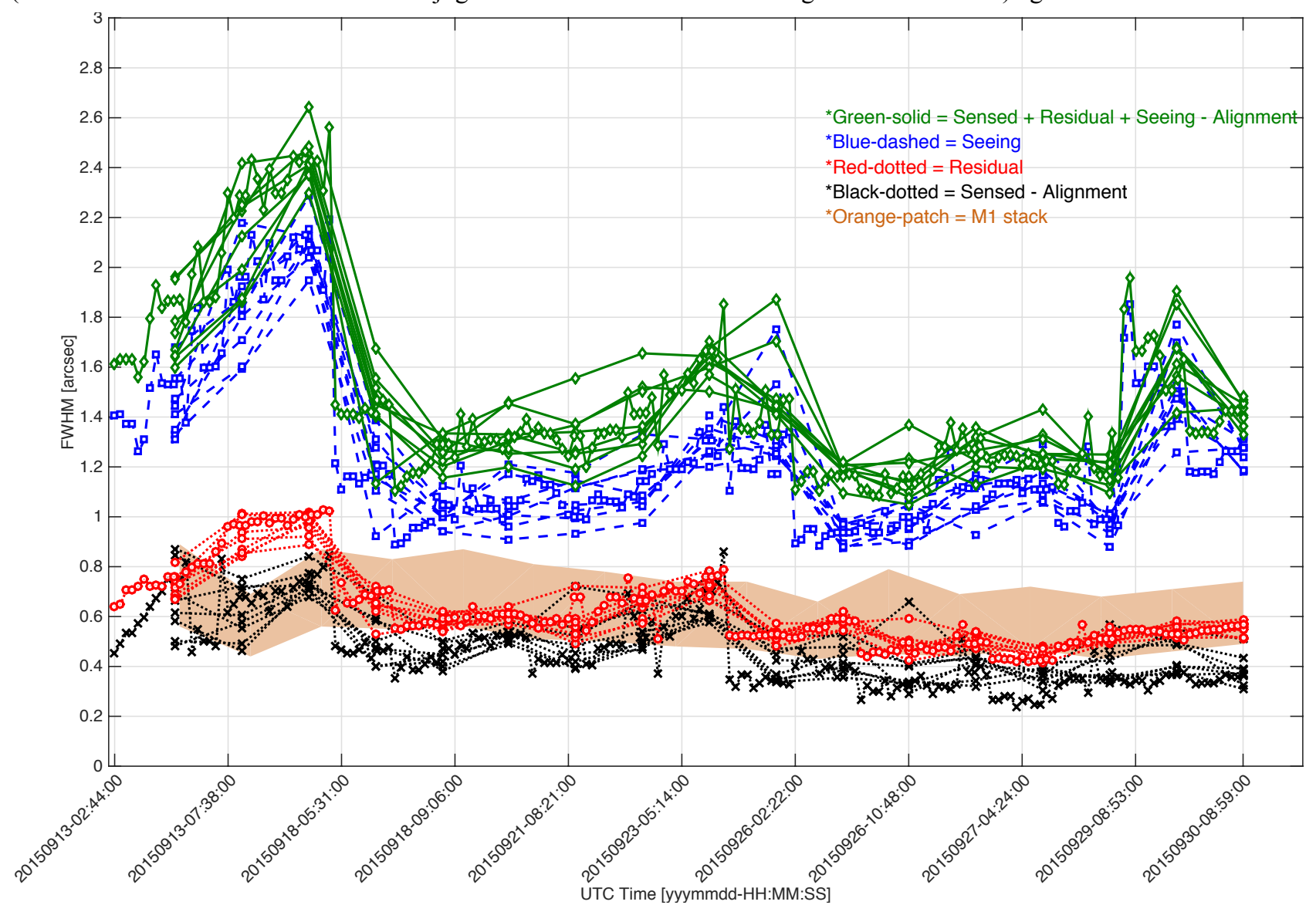

Figure 24 The image quality estimate in term of the PSF FWHM based on the DWFS data.

The DWFS data can be utilized to determine not only the field aberrations, but also the image quality of the telescope in terms of the PSF FWHM. The slope deviations across the telescope apertures can be considered as lateral ray aberrations and they can be used to calculate the geometric FWHM of a PSF. The DWFS data can also be decomposed into three different components. The first one corresponds to the aberration modes that can be completely captured by the DWFS sensors given their sub-aperture geometries. In this case, these are the first 55 annular Zernike modes. The second component corresponds to those modes that cannot be sensed by the DWFS, which are basically all other contributions from the Zernike modes beyond those sensed. Some of this un-sensed higher-order contribution can alias into the sensed low order contribution, but most of it manifests itself in the form of residual slope deviation after subtracting out the sensed portion from the data. The last component has no direct contribution to the measured slope deviation, but shows up as the size of the PSFs in the individual sub-apertures. The DWFS exposure time was set to a value between 15 to 60 seconds in order to average out the sub-aperture PSF motion as much as possible and thus each sub-aperture essentially captures the seeing information in its PSF. Thus, by measuring the FWHM of the individual subaperture PSFs, one can estimate the seeing contribution at the time of the measurement. One caveat of this approach is that the sub-aperture size must have been matched to that of the isoplanatic patch size (i.e. Fried constant). In the DWFS, the sub-aperture size was approximately $5 \sim 8$ times larger than the isoplanatic patch size. Therefore, the seeing estimate 
from the sub-aperture PSF data should be treated as an approximation. In this context, Figure 24 displays these components as functions of time. The black-dotted curves show the FWHM contributed by the sensed modes across all DWFS. Note that, in the sensed contribution, we have subtracted the offset contributions from the WFC's rigid-body alignment error with respect to M1 because this effect can be compensated in the actual operation. The red-dotted curves correspond to the residual FWHM contributions of all DWFS. The orange-colored patch marks the range of the M1 stacking quality in terms of the FWHM contribution. The blue-dashed curves indicate the seeing contributions estimated by taking the median value across the sub-aperture PSFs of each DWFS. The green-solid curves show the total FWHM of the telescope PSFs at each DWFS field location and are computed by the root-sum-square of all contributions. As one can see easily, there is an interesting correlation between the sensed, residual, and seeing components that clearly indicates the aliasing effects. This effect is rather visible in the early data set and this turned out to be due to that in those data sets the DWFS exposure time was rather short ( 5 to 7 seconds). Thus the seeing effect seems correlated strongly with the residual contribution but rather weakly with the sensed contribution. This trend seems occurring in other data sets with much longer DWFS exposure times, but the degree of correlation seems at a much low-level. Another observation of this data is that the residual contribution appears to show rather strong correlation with the M1 stacking quality, which confirmed our initial expectation that the residual contribution would come from the M1 stacking error because a large fraction of the segment tip/tilt error happens in the spatial scale beyond what the DWFS can sense. Based on the DWFS data analysis, we conclude that the HET with the WFC system is capable of producing a PSF FWHM between 0.3 and 0.8 arcsec over the science field of view. The unsensed contribution, mainly from the M1 stacking quality, varies in the PSF FWHM from 0.4 to 1 arcsec. The seeing contribution as estimated by the DWFS is between 0.9 and 2.2 arcsec with median 1.2 arcsec. The total PSF FWHM is estimated to be between 1.0 and 2.6 arcsec with median 1.36 arcsec across the field. The telescope imaging performance was declared to be adequate for the science instrument commissioning and operation in October 2 of 2015.

\section{CONCLUSION}

In this paper, we summarized the construction, delivery, installation, and on-sky verification of the WFC system for the upgraded HET. Like any other mid-to-large scale astronomical instrumentation projects, we faced technical challenges during this project, but these challenges were uncovered in the very late stage of the project and their nature turned out to be severely ill defined. We paid a special attention to these challenges and the ways we responded to overcome those. We then highlighted the aspects of the WFC transportation from Tucson AZ to the HET in Fort Davis TX and described the subsequent on-site verification tests that confirmed the safe transportation of the system. Finally, we described the optical alignment of the three major telescope subsystems, namely the PFIP, the WFC, and the M1 and the subsequent month-long multiple field wavefront sensing campaign to verify the alignment of the WFC and to evaluate the imaging performance of the full telescope system. As discussed, we had many challenges and struggles in delivering this system during the past 7 years, many talented people's ingenuities came together to overcome those difficulties and led to a highly performing imaging system on a 10-meter class telescope. At the time of this writing, the telescope is capable of efficiently pointing, guiding, and tracking stars with stable image quality and two science instruments are being actively commissioned, i.e. the VIRUS and the LRS2 instrument. We look forward to the remaining two facility instruments (the High Resolution Spectrograph and the Habitable-Zone Planet Finder) coming online soon.

\section{ACKNOWLEDGEMENTS}

HETDEX is run by the University of Texas at Austin McDonald Observatory and Department of Astronomy with participation from the Ludwig-Maximilians-Universität München, Max-Planck-Institut für Extraterrestriche-Physik, Leibniz-Institut für Astrophysik Potsdam (AIP), Texas A\&M University, Pennsylvania State University, Institut für Astrophysik Göttingen, University of Oxford, Max-Planck-Institut für Astrophysik and The University of Tokyo. In addition to Institutional support, HETDEX is funded by the National Science Foundation (grant AST-0926815), the State of Texas, the US Air Force (AFRL FA9451-04-2-0355), by the Texas Norman Hackerman Advanced Research Program under grants 003658-0005-2006 and 003658-0295-2007, and by generous support from private individuals and foundations. We would like to express our gratitude to Dan Fabricant and Fred Hearty for their critical review on the readiness of the WFC. Hanshin Lee is much grateful for the continuous strong support from his family Olivia and Sedona throughout the project. 


\section{REFERENCES}

[1] Hill, G.J., Drory, N., Good, J.M., Lee, H., Vattiat, B.L., Kriel, H., Ramsey, J., Randy Bryant, R., Elliot, L., Fowler, J., Landiau, M., Leck, R., Odewahn, S., Perry, D., Savage, R., Schroeder Mrozinski, E., Shetrone, M., Damm, G., Gebhardt, K., MacQueen, P.J., Martin, J., Armandroff, T., Ramsey, L.W., "The Hobby-Eberly Telescope wide-field upgrade", Proc. SPIE 9906-5 (2016).

[2] Burge, J.H., Benjamin, S.D., Dubin, M.B., Manuel, S.M., Novak, M.J., Oh, C.J., Valente, M.J., Zhao, C., Booth, J.A., Good, J.M., Hill, G.J., Lee, H., MacQueen, P.J., Rafal, M.D., Savage, R.D., Smith, M.P., and Vattiat, B.L., "Development of a wide-field spherical aberration corrector for the Hobby Eberly Telescope", Proc. SPIE, 7733-51 (2010)

[3] Hill, G.J., Tuttle, S.E., Vattiat, B.L., Lee, H., Drory, N., Kelz, A., Ramsey, J., DePoy, D.L., Marshall, J.L., Gebhardt, K., Chonis, T.S., Dalton, G.B., Farrow, D., Good, J.M., Haynes, D.M., Indahl, B.L., Jahn, T., Kriel, H., Montesano, F., Nicklas, H., Noyola, E., Prochaska, T., Allen, R.D., Blanc, G., Fabricius, M.H., Landriau, M., MacQueen, P.J., Roth, M.M., Savage, R., Snigula, J.M., "VIRUS: first deployment of the massively replicated fiber integral field spectrograph for the upgraded Hobby-Eberly Telescope", Proc. SPIE, 9908-54 (2016)

[4] Chonis, T.S., Hill, G.J., Lee, H., Tuttle, S.E., Vattiat, B.L., Drory, N., Indahl, B.L., Peterson, T.W., Ramsey, J., "LRS2 - design, assembly, testing, and commissioning of the second generation low resolution spectrograph for the Hobby-Eberly Telescope”, Proc. SPIE 9908-163 (2016).

[5] Vattiat, B., Lee, H., Chonis, T., Hill, G.J., Haubnitz-Reinke, C. "Design and Assembly of the Low Resolution Spectrograph 2 Integral Field Unit," Proc. SPIE 9908-138 (2016).

[6] Tuttle, S., et al., "VIRUS early installation and commissioning", Proc. SPIE, 9908-55 (2016).

[7] Hill, G.J., "HETDEX and VIRUS: Panoramic Integral Field Spectroscopy with 35k fibres" in 'Multi-Object Spectroscopy in the Next Decade' a conference held in La Palma, March 2015, (eds. I. Skillen, M. Balcells \& S. Trager), ASP Conference Series, in press (2016).

[8] Hanshin Lee, "Optimal collimation of misaligned optical systems by concentering primary field aberrations," Opt. Express 18, 19249-19262 (2010).

[9] R. V. Shack and K. Thompson, "Influence of alignment errors of a telescope system on its aberration field," in Optical alignment, R. M. Shagam and W. C. Sweatt, eds., Proc. SPIE251, 146-153 (1980).

[10]B. McLeod, "Collimation of Fast Wide-Field Telescopes," Publ. Astron. Soc. Pac. 108, 217-219 (1996).

[11]R. N. Wilson and B. Delabre, "Concerning the Alignment of Modern Telescopes: Theory, Practice, and Tolerance Illustrated by the ESO NTT," Publ. Astron. Soc. Pac. 109, 53-60 (1997).

[12] H. Lee, G. Dalton, I. Tosh, and S. Kim, "Computer-guided alignment III: Description of inter-element alignment effect in circular-pupil optical systems," Opt. Express 16, 10992-11006 (2008).

[13] John M. Good, Gary J. Hill, Emily Schroeder-Mrozinski, Richard Savage, Herman Kriel, Scott Benjamin, Robert Stone, "Performance of Cable Isolators in the Transport of Large Optical Assemblies", Proc. SPIE 9906-14 (2016).

[14] Vattiat, B.L., Hill, G.J., Lee, H., Moreira, W., Drory, N., Ramsey, J., Elliot, L., Landriau, M., Perry, D.M., Savage, R., Kriel, H., Haeuser, M., and Mangold, F., "Design, alignment, and deployment of the Hobby Eberly Telescope prime focus instrument package", Proc. SPIE, 9147-172 (2014).

[15] H. Lee, et al., "Metrology systems of Hobby-Eberly Telescope wide field upgrade," Proc. SPIE, 8444-181 (2012). 